\title{
Álgebras de Tipo Mesh Fortemente Simplesmente Conexas
}

\author{
Rosana Retsos Signorelli Vargas
}

TESE APRESENTADA

$\mathrm{AO}$

INSTITUTO DE MATEMÁTICA E ESTATÍSTICA

DA

UNIVERSIDADE DE SÃO PAULO

PARA OBTENÇÃO DO GRAU

DE

DOUTORA EM MATEMÁTICA

Área de Concentração: Álgebra
Orientador: Prof. Dr. Flávio Ulhoa Coelho

Durante a elaboração deste trabalho

a autora recebeu apoio financeiro do CNPq.

-São Paulo, abril de 1999- 


\section{ÁLGEBRAS DE TIPO MESH FORTEMENTE SIMPLESMENTE CONEXAS}

Este exemplar corresponde à redação final da tese devidamente corrigida e defendida por

Rosana Retsos Signorelli Vargas e aprovada pela Comissão Julgadora.

São Paulo, 08 de julho de 1999.

Banca examinadora:

- Prof. Dr. Flávio Ulhoa Coelho

- Prof. Dr. Héctor Alfredo Merklen Goldschmidt

- Prof. Dr. Antonio Paques

- Prof. Dr. Miguel Angel Alberto Ferrero

- Prof. Dr. Alfredo Rosário Jones Rodrigues 
Este trabalho é dedicado aos meus pais, José Antônio e Georgia. $E$ ao meu esposo, Edson. 
O mistério das coisas, onde está ele?

Onde está ele que não aparece

Pelo menos a mostrar-nos que é mistério?

Que sabe o rio disso e que sabe a árvore?

E eu, que não sou mais do que eles, que sei disso?

Sempre que olho para as coisas e penso no que os homens

pensam delas,

Rio como um regato que soa fresco numa pedra.

Porque o único sentido oculto das coisas

É elas não terem sentido oculto nenhum,

É mais estranho do que todas as estranhezas

$E$ do que os sonhos de todos os poetas

E os pensamentos de todos os filósofos,

Que as coisas sejam realmente o que parecem ser

E não haja nada que compreender.

Sim, eis o que os meus sentidos aprenderam sozinhos:-

As coisas não têm significação: têm existência.

As coisas são o único sentido oculto das coisas.

Fernando Pessoa. 


\section{Abstract}

The purpose of this work is to study the following class of algebras. Let $\Delta$ be a finite translation quiver and $k$ be an algebraically closed field. The algebra given by the quotient of $k \Delta$ by the ideal generated by its mesh relations is called the mesh algebra of $\Delta$. Such a class of algebras include the hereditary and the Auslander algebras.

Having in mind the Auslander algebras, we shall study here the mesh algebras concerning their homological properties, as well as its representation type, one-point extensions, existence of postprojective components.

As a main result, we shall obtain a characterization of mesh algebras which are strongly simply connected in terms of the Hochschild cohomology, the separation condition, irreducible contours and orbit-graphs.

\section{Resumo}

O objetivo deste trabalho é estudar a seguinte classe de álgebras. Seja $\Delta$ uma aljava finita, com translação e $k$ um corpo algebricamente fechado. A álgebra dada pelo quociente de $k \Delta$ pelo ideal gerado por suas relações de tipo mesh é chamada de álgebra de tipo mesh de $\Delta$. Tal classe de álgebras inclui as álgebras hereditárias e as de Auslander.

Tendo em mente as álgebras de Auslander, iremos estudar aqui as álgebras de tipo mesh do ponto de vista de suas propriedades homológicas, assim como seu tipo de repesentação, extensões por um ponto, existência de componentes pós-projetivas.

Como resultado principal obtivemos uma caracterização das álgebras de tipo mesh que são fortemente simplesmente conexas em termos da cohomologia de Hochschild, condição de separação, contornos irredutíveis e grafos-órbitas. 


\section{Agradecimentos}

A gratidão é uma partilha,...

Gostaria de agradecer a todos que de alguma maneira participaram e colaboraram para que este trabalho fosse realizado.

Ao CNPq pelo apoio financeiro.

Aos professores do Instituto de Matemática e Estatística da USP que contribuíram para minha formação matemática.

Ao grupo de representações de álgebra deste Instituto que trabalha de maneira efetiva proporcionando uma atmosfera científica rica e estimulante. Aos professores Hector, Eduardo e Maria Izabel, obrigada pelo incentivo e pela disposição em ajudar. À Gladys, pela sua generosidade, sempre pronta a esclarecer alguma dúvida ou a indicar referências bibliográficas interessantes.

Ao meu orientador, Flávio Coelho, pela sua dedicação. Por ter sugerido o contexto específico no qual trabalhamos bem como alguns problemas que resultaram neste trabalho de tese. Pelas inúmeras discussões e comentários que contribuíram para o melhor entendimento deste trabalho e que refletiram também em meu amadurecimento matemático em geral.

Ao colega Cristian com quem pude falar, falar... e escutar, escutar... sobre várias questões matemáticas no tempo em que este trabalho ainda era um esboço. À todos os amigos do IME que tornaram o dia a dia acadêmico mais humano e divertido. Ao Paulo, pelas dicas sobre Emacs durante a digitação deste trabalho.

Em especial aos meus queridos pais que sempre me incentivaram a buscar o caminho do conhecimento. E que juntamente com meu irmão Carlos formam um forte suporte emocional familiar onde posso buscar o equilíbrio.

Ao Edson, meu companheiro de todas as horas... e quando parecia que eu estava sozinha e que as idéias voavam para longe, lá estava o Edson ao meu lado. Obrigada pelo seu otimismo, carinho e compreensão que foram essenciais para que eu pudesse vencer os obstáculos desta fase .

..." a gratidão é como um eco de alegria." Por isso quero compartilhar com todos vocês a minha alegria pela conclusão deste trabalho. 
Acendemos paixões no rastilho do próprio coração. O que amamos é sempre chuva, entre o vôo da nuvem e a prisão do charco. Afinal, somos caçadores que a si mesmo se azagaiam. No arremesso certeiro vai sempre um pouco de quem dispara.

Mia Couto. 


\section{Sumário}

Introdução

1 Conceitos e resultados preliminares 1

1.1 Preliminares ........................... 1

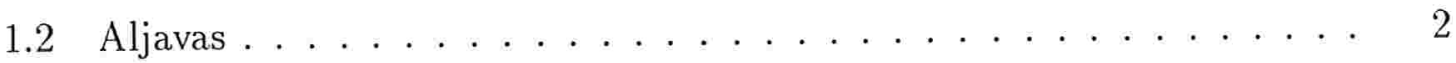

1.3 Grafos-órbitas . . . . . . . . . . . . . . . 5

1.4 Álgebras de caminhos . . . . . . . . . . . . . . . . 8

1.5 Módulos e representações de álgebras . . . . . . . . . . . . . . . . 10

1.6 Sequências e aljavas de Auslander-Reiten . . . . . . . . . . . . . . 12

1.7 Componentes pós-projetivas . . . . . . . . . . . . 16

1.8 Extensão por um ponto de álgebras . . . . . . . . . . . . . . . 18

1.9 Cohomologia de Hochschild . . . . . . . . . . . . . . . 20

1.10 Álgebras simplesmente conexas . . . . . . . . . . . . . . . . 22

2 Álgebras de tipo mesh $\quad 28$

2.1 Álgebras de Auslander . . . . . . . . . . . . . . . . . . . 29

2.2 Álgebras de tipo mesh e exemplos . . . . . . . . . . . . . . . 31 
2.3 Algumas propriedades para as álgebras de tipo mesh . . . . . . . 34

$2.4 \mathrm{Um}$ caso particular de álgebras de tipo mesh . . . . . . . . . . . 38

3 Álgebras de tipo mesh fortemente simplesmente conexas em característica zero

3.1 Cohomologia de Hochschild para álgebras de tipo mesh . . . . . . . . 48

3.2 Ciclos e contornos para álgebras de tipo mesh . . . . . . . . . 52

3.3 Demonstração do resultado principal . . . . . . . . . . . . 63

3.4 Consequências e exemplos . . . . . . . . . . . . . . . . 64

$\begin{array}{lr}\text { Bibliografia } & 65\end{array}$ 


\section{Introdução}

O principal objetivo da teoria de representações de álgebras é estudar álgebras via o estudo de suas categorias de módulos. Em um contexto mais geral estuda-se a categoria dos módulos finitamente gerados à direita (ou à esquerda) sobre uma álgebra de Artin. Tal álgebra de Artin é um anel artiniano finitamente gerado sobre o seu centro que é um anel artiniano comutativo. Estaremos considerando neste trabalho um contexto mais particular que é o das álgebras de dimensão finita sobre um corpo algebricamente fechado.

Um dos problemas clássicos na teoria de representações é descobrir qual é o tipo de representação de uma álgebra. Este problema consiste em saber se o número de classes de isomorfia de módulos indecomponíveis sobre a álgebra é finito ou infinito.

No estudo das álgebras de tipo de representação finito foi considerada uma outra classe de álgebras de artin chamada álgebras de Auslander. Uma álgebra de Auslander é uma álgebra de endomorfismos $A=\operatorname{End}_{\Lambda}\left(\bigoplus_{i=1}^{d} M_{i}\right)$ onde $\Lambda$ é uma álgebra de tipo de representação finito e $\left\{M_{1}, \ldots, M_{d}\right\}$ é um conjunto completo de representantes de classes de isomorfia de $\Lambda$-módulos indecomponíveis. As álgebras de Auslander foram introduzidas primeiro em [9] e são caracterizadas pelas suas propriedades homológicas, que são úteis, por exemplo, no desenvolvimento da teoria de recobrimento. Estas álgebras foram a motivação do nosso principal objeto de estudo neste trabalho: as álgebras de tipo mesh.

Uma álgebra de tipo mesh é uma álgebra de caminhos de uma aljava finita e com translação $\Delta$ sobre um corpo algebricamente fechado módulo as relações de tipo 
mesh ( para mais detalhes ver seção 2.2).

Em uma primeira parte do trabalho investigamos algumas questões a respeito das álgebras de tipo mesh. Ao trabalharmos com estas álgebras estamos em um contexto mais geral que o das álgebras de Auslander. Lembramos que as álgebras de Auslander têm dimensão global no máximo 2. Esta limitação, porém, não existe para álgebras de tipo mesh, na realidade exibimos exemplos de álgebras de tipo mesh com dimensão global arbitrariamente grande, inclusive infinita. A seguir, tratamos de um caso particular de álgebras de tipo mesh definida a partir da partição pós-projetiva de uma álgebra e, neste caso particular as dimensões globais estão efetivamente limitadas por 2. Além disso, iremos dar uma condição necessária para que estas últimas álgebras sejam de tipo finito.

Na segunda parte do trabalho temos como enfoque principal a caracterização das álgebras de tipo mesh fortemente simplesmente conexas que é uma classe de álgebras simplesmente conexas. No estudo das álgebras de tipo de representação finito, Bongartz e Gabriel [16] introduziram em 1982 o conceito das álgebras simplesmente conexas e as caracterizaram pela propriedade de separação. Este conceito, juntamente com as técnicas de recobrimento, desempenhou um importante papel no entendimento das álgebras de tipo de representação finito (ver, por exemplo [15], [18]). Um pouco mais tarde, Assem e Skowroński [3] estenderam a noção de álgebras simplesmente conexas para as álgebras de tipo de representação infinito. A classe de álgebras simplesmente conexas que estamos tratando de modo especial neste trabalho que é a das álgebras fortemente simplesmente conexas foi introduzida por Skowroński em [42]. Neste mesmo artigo Skowroński as caracterizou pela propriedade de separação e pelo anulamento do primeiro grupo de cohomologia de Hochschild. Ultimamente, o estudo das álgebras fortemente simplesmente conexas tem atraído o interesse de muitos pesquisadores ( [5], [6], [7], [8]) e alguns progressos também têm sido feitos no caso manso ( [38], [43]). A caracterização que obtivemos das álgebras de tipo mesh fortemente simplesmente conexas relaciona os conceitos já mencionados de cohomologia de Hochschild, condição de separação e outros como aljavas-órbitas, grafos-órbitas e propriedades sobre a aljava ordinária da álgebra. Esta última pro- 
priedade foi baseada em uma formalização adotada por Assem e Liu em [7] e pode ser verificada facilmente examinando a aljava ordinária da álgebra. Com isto, temos um critério para reconhecer diretamete a partir de sua aljava quando uma álgebra de tipo mesh é fortemente simplesmente conexa ou não.

Quanto ao conceito de Cohomologia de Hochschild, Happel mostrou em [33] que uma álgebra $\Lambda$ de tipo de representação finito é simplesmente conexa se e somente se o primeiro grupo de cohomologia de Hochschild $H^{1}(A)$ se anula (onde $A$ é a álgebra de Auslander de $\Lambda$ ). Posteriormente, Assem e Brown [5] generalizaram o resultado de Happel, acrescentando mais algumas equivalências. Uma motivação para esta generalização foi a de encontrar um critério para a simples conexidade de uma classe de álgebras, sendo a maior parte delas de tipo de representação infinito, já que pouco se sabe sobre técnicas de recobrimento no caso de representação infinito. Daí o interesse em estudar problemas de descrição de álgebras de tipo de representação infinto simplesmente conexas. Por isso também tivemos interesse em caracterizar as álgebras de tipo mesh fortemente simplesmente pois podemos visualizar outros exemplos de álgebras simplesmente conexas de tipo de representação infinito além das já conhecidas álgebras inclinadas de tipos Euclidianos $\tilde{\mathbf{D}}_{n}, \tilde{\mathbf{E}}_{p}(n \geq 4, p=6,7,8)$, as tubulares de Ringel e das álgebras inclinadas mansas fortemente simplesmente conexas que foram caracterizadas recentemente por Assem, Liu e de la Peña [8].

Outro ponto que gostaríamos de ressaltar é que as álgebras fortemente simplesmente conexas possuem componentes pós-projetivas pois satisfazem a condição de separação. Nesta direção podemos exibir várias álgebras de tipo mesh que possuem componentes pós-projetivas. Por outro lado, existem álgebras álgebras de tipo mesh que não possuem componentes pós-projetivas (ver exemplos na seção 2.3). No caso das álgebras de Auslander conjectura-se a existência de componentes pós-projetivas. A importância de uma álgebra possuir componentes pós-projetivas está no fato de que se um módulo $X$ está em uma componente pós-projetiva ele tem certas propriedades como: ele é homologicamente trivial, isto é, $\operatorname{Ext}_{\Lambda}^{i}(X, X)=0$ para $i>0, \operatorname{End}_{\Lambda} X=k$ e sua classe de isomorfia é unicamente determinada por seus fatores de composição. 
O nosso trabalho está organizado da seguinte maneira:

Em uma primeira parte do Capítulo 1 apresentamos os conceitos básicos da teoria. Depois direcionamos o estudo para um contexto mais específico com o qual estamos interessados, apresentando os conceitos de cohomologia de Hochschild, álgebras simplesmente conexas, álgebras fortemente simplesmente conexas e os resultados que os caracterizam e que os relacionam em alguns casos.

No Capítulo 2, apresentamos as álgebras de Auslander e alguns resultados principais envolvendo as mesmas. Logo depois, introduzimos o conceito de álgebras de tipo mesh que é o nosso principal objeto de estudo neste trabalho. Nesta direção discutimos propriedades que as álgebras de tipo mesh satisfazem utilizando-as como parâmetros de comparação com as álgebras de Auslander. Ao final do Capítulo 2 analisamos um exemplo de álgebras de tipo mesh que são definidas a partir de partições pós-projetivas.

No Capítulo 3 apresentamos os conceitos de ciclos, contornos, redutibilidade e contratilidade muito úteis na formalização de propriedades sobre as aljavas. Enunciamos e demonstramos o resutado principal e analisamos algumas consequências do mesmo. 


\section{Capítulo 1}

\section{Conceitos e resultados preliminares}

\subsection{Preliminares}

Em todo trabalho estaremos considerando $\Lambda$ uma $k$-álgebra de dimensão finita sobre um corpo $k$ algebricamente fechado, associativa e com identidade. Representamos por $\bmod \Lambda$ a categoria cujos objetos são os $\Lambda$-módulos finitamente gerados à direita e cujos morfismos são os morfismos entre módulos. Vamos denotar por ind $\Lambda$ a subcategoria de $\bmod \Lambda$ com um representante de cada classe de isomorfia de módulos indecomponíveis.

Temos que todo $\Lambda$-módulo finitamente gerado é soma direta finita de $\Lambda$ módulos finitamente gerados indecomponíveis e tal decomposição é sempre única, salvo isomrfismos. Seja $\Lambda=P_{1}^{s_{1}} \oplus \cdots \oplus P_{t}^{s_{t}}$ a decomposição de $\Lambda$ em $\Lambda$-módulos indecomponíveis onde $P_{i}$ não é isomorfo a $P_{j}$ se $i \neq j$. Quando $s_{i}=1$ para todo $i=1, \ldots, t$ dizemos que $\Lambda$ é uma álgebra básica.

Na teoria de representações de álgebras o principal objetivo é estudar a categoria $\bmod \Lambda$. Usando o Teorema de Morita podemos restringir o estudo às categorias de módulos sobre álgebras básicas e indecomponíveis.

Neste Capítulo daremos um apanhado dos conceitos e resultados principais 
para o entendimento deste trabalho. Como referências para as seções 1.2, 1.3, 1.4 e 1.5 citamos [13], [20], [22].

\subsection{Aljavas}

Uma aljava $\Delta$ é dada por dois conjuntos $\Delta_{0}$ e $\Delta_{1}$, onde os elementos de $\Delta_{0}$ são chamados de vértices e os elementos de $\Delta_{1}$ são chamados de flechas e duas aplicações

$$
\begin{aligned}
& s: \Delta_{1} \longrightarrow \Delta_{0} \quad, \quad e: \Delta_{1} \longrightarrow \Delta_{0} \\
& \alpha \longmapsto x \quad \alpha \longmapsto y
\end{aligned}
$$

que indicam os vértices inicial e final da flecha $\alpha: x \rightarrow y$.

Exemplos de aljavas:

Exemplo 1.1.

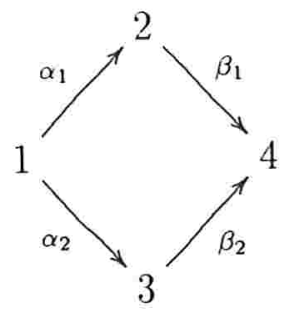

$$
\begin{aligned}
& \{1,2,3,4,5,6\}=\Delta_{0} \\
& \left\{\alpha_{1}, \alpha_{2}, \beta_{1}, \beta_{2}, \delta, \theta\right\}=\Delta_{1} \\
& s\left(\alpha_{1}\right)=s\left(\alpha_{2}\right)=1 \\
& s\left(\beta_{1}\right)=e\left(\alpha_{1}\right)=2 \\
& s\left(\beta_{2}\right)=e\left(\alpha_{2}\right)=3 \\
& e\left(\beta_{1}\right)=e\left(\beta_{2}\right)=4 \\
& s(\gamma)=5 \\
& e(\delta)=s(\theta)=e(\theta)=6 .
\end{aligned}
$$


Exemplo 1.2.

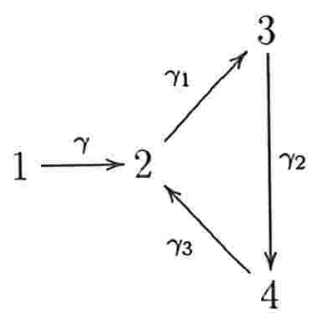

$$
\begin{aligned}
& \{1,2,3,4\}=\Delta_{0} \\
& \left\{\gamma, \gamma_{1}, \gamma_{2}, \gamma_{3}\right\}=\Delta_{1} \\
& s(\gamma)=1 \\
& s\left(\gamma_{1}\right)=e\left(\gamma_{3}\right)=e(\gamma)=2 \\
& s\left(\gamma_{2}\right)=e\left(\gamma_{1}\right)=3 \\
& s\left(\gamma_{3}\right)=e\left(\gamma_{2}\right)=4
\end{aligned}
$$

\section{Exemplo 1.3.}

$$
\begin{array}{ll} 
& \{1,2,3,4,5\}=\Delta_{0} \\
& \left\{\alpha_{1}, \alpha_{2}, \alpha_{3}, \alpha_{4}\right\}=\Delta_{1} \\
& s\left(\alpha_{2}\right)=1 \\
& s\left(\alpha_{1}\right)=2 \\
& s\left(\alpha_{3}\right)=e\left(\alpha_{2}\right)=3 \\
& s\left(\alpha_{4}\right)=e\left(\alpha_{1}\right)=e\left(\alpha_{3}\right)=4 \\
& e\left(\alpha_{4}\right)=5
\end{array}
$$

Um grafo $\bar{\Delta}$ é uma aljava sem orientações, isto é, onde as flechas são substituidas por arestas.

Um caminho $\gamma$ na aljava $\Delta$ é definido da seguinte forma: $\gamma=\alpha_{n} \ldots \alpha_{1}$ onde para cada $1 \leq i \leq n-1, e\left(\alpha_{i}\right)=s\left(\alpha_{i+1}\right)$. O número de flechas neste caminho $\gamma(=n)$ é o comprimento de $\gamma$ e será denotado por $l(\gamma)$. Um caminho de comprimento zero (ou um caminho trivial) é um caminho sem flechas associado a um vértice $a \in \Delta_{0}$ e que denotamos por $e_{a}$. No exemplo 1.1 acima temos seis caminhos triviais $e_{1}, e_{2}, e_{3}, e_{4}, e_{5}, e_{6}$, um para cada vértice.

Dado um caminho $\gamma=\alpha_{n} \ldots \alpha_{1}$ denotamos por $s(\gamma)=s\left(\alpha_{1}\right)$ o vértice inicial de $\gamma$ e por $e(\gamma)=e\left(\alpha_{n}\right)$ o vértice final de $\gamma$. No caso em que $\gamma$ não é um caminho 
trivial e $s(\gamma)=e(\gamma)$ dizemos que $\gamma$ é um circuito orientado. Quando $l(\gamma)=1$ e $s(\gamma)=e(\gamma)$ dizemos que $\gamma$ é um laço. Uma aljava dirigida é uma aljava que não possui circuitos orientados. No exemplo 1.2 aparece o circuito orientado $\gamma_{3} \gamma_{2} \gamma_{1}$ e no exemplo 1.1, $\theta$ é um laço. Nos exemplos acima apenas a aljava do exemplo $\mathbf{1 . 3}$ é uma aljava dirigida. Um passeio entre os vértices $a$ e $b$ é dado por uma sequência de flechas $\alpha_{1}, \ldots, \alpha_{n}$ onde $a \in\left\{s\left(\alpha_{1}\right), e\left(\alpha_{1}\right)\right\}, b \in\left\{s\left(\alpha_{n}\right), e\left(\alpha_{n}\right)\right\}$ e para $1 \leq i \leq n-1$, $\alpha_{i}$ e $\alpha_{i+1}$ têm um vértice em comum.

Uma aljava $\Delta$ é dita conexa quando dados quaisquer dois vértices $x$ e $y$ em $\Delta_{0}$, existe algum passeio entre eles. Nos exemplos 1.2 e 1.3 as aljavas são conexas enquanto que no exemplo 1.1 a aljava não é conexa.

Uma subaljava da aljava $\Delta=\left(\Delta_{0}, \Delta_{1}, s, e\right)$ é uma aljava $\Delta^{\prime}=\left(\Delta_{0}^{\prime}, \Delta_{1}^{\prime}, s^{\prime}, e^{\prime}\right)$ onde $\Delta_{0}^{\prime} \subset \Delta_{0}, \Delta_{1}^{\prime} \subset \Delta_{1}$ e as restrições de $s$ e $e$ a $\Delta_{1}^{\prime}$ são iguais respectivamente a $s^{\prime}$ e $e^{\prime}$. Uma subaljava $\Delta^{\prime}$ de $\Delta$ é dita plena se para cada flecha $\alpha: a \rightarrow b \in \Delta_{1}$ com vértices $a, b \in \Delta_{0}^{\prime}$ então $\alpha \in \Delta_{1}^{\prime}$.

Uma aljava de tipo árvore é uma aljava que não possui subaljava do tipo $\tilde{\mathbf{A}}_{n}$ : $1 \longrightarrow \quad \cdots \quad \bullet-n-n+1=1$ com $n \geq 2$. Dos exemplos anteriores apenas o exemplo $\mathbf{1 . 3}$ é um exemplo de aljava de tipo árvore.

Uma aljava $\Delta$ é dita com translação quando :

- $\Delta$ for localmente finita (isto é, cada vértice de $\Delta_{0}$ é o vértice inicial e final de apenas um número finito de flechas);

- $\Delta$ não tiver laços ;

- Existir uma aplicação bijetora $\begin{aligned} \tau: \widehat{\Delta}_{0} & \longrightarrow \widehat{\widehat{\Delta}}_{0} \\ x & \longmapsto \tau x\end{aligned}$ onde $\widehat{\Delta}_{0}$ e $\widehat{\widehat{\Delta}}_{0}$ são subconjuntos de $\Delta_{0}$, tal que se $x \in \widehat{\Delta}_{0}$ então para todo $y \in \Delta_{0}$, o número de flechas de $y$ para $x$ é igual ao número de flechas de $\tau x$ para $y$.

Observamos que em particular nós temos a propriedade $x^{-}=(\tau x)^{+}$, onde $x^{-}=\{y \in$ $\Delta_{0} / \exists$ uma flecha $\left.y \rightarrow x\right\}$ e $(\tau x)^{+}=\left\{y \in \Delta_{0} / \exists\right.$ uma flecha $\left.\tau x \rightarrow y\right\}$. Esta proprieda- 
de também é suficiente no caso em que não existem flechas múltiplas na aljava $\Delta$. Vamos denotar $\tau^{0} x$ como sendo o próprio $x$.

Um vértice $x \in \Delta_{0} \backslash \widehat{\Delta}_{0}$ será chamado de vértice projetivo e neste caso denotamos $\tau(x)=0$.

Um vértice $x \in \Delta_{0} \backslash \widehat{\widehat{\Delta}}_{0}$ será denominado vértice injetivo e neste caso denotamos $\tau^{-1}(x)=0$.

Dada uma aljava com translação $\Delta=\left(\Delta_{0}, \Delta_{1}, \tau\right)$, uma semitranslação de $\Delta$ é dada por uma aplicação bijetora $\begin{array}{rlll}\sigma: & \tilde{\Delta}_{1} & \longrightarrow & \tilde{\tilde{\Delta}}_{1} \\ \alpha & \longmapsto & & \\ & \alpha(\alpha)\end{array}$ onde $\tilde{\Delta}_{1}$ e $\tilde{\Delta}_{1}$ são subconjuntos de $\Delta_{1}$, tal que se a flecha $\alpha: y \rightarrow x$ está em $\tilde{\Delta}_{1}$ então $x$ é um vértice não projetivo, tal que $\sigma(\alpha): \tau x \rightarrow y$.

No caso em que $\Delta$ não possui flechas múltiplas, existe uma única semitranslação. Quando temos flechas múltiplas observamos que podemos ter em geral mais de uma maneira de definir a semitranslação. Mas, sem perda de generalidade, podemos fixar uma delas.

Um vértice $x \in \Delta_{0}$ será chamado de fonte em $\Delta$ quando $x^{-}$for um conjunto vazio em $\Delta$.

Um vértice $x \in \Delta_{0}$ será chamado de poço em $\Delta$ quando $x^{+}$for um conjunto vazio em $\Delta$.

\subsection{Grafos-órbitas}

Sejam $\Delta$ uma aljava finita (isto é, os conjuntos $\Delta_{0}$ e $\Delta_{1}$ são finitos), dirigida. com translação e $x \in \Delta_{0}$. A $\tau$-órbita de $x$ é o conjunto $o(x)$ consistindo de todos $\tau^{n}(x)$ definidos, com $n \in \mathbb{Z}$. Observe que para cada $x \in \Delta_{0}$, a sua $\tau$-órbita $o(x)$ possui um único vértice projetivo $p_{x}$ e um único vértice injetivo $i_{x}$. De fato, como a aljava $\Delta$ é finita e dirigida devem existir $n_{1}>0$ e $n_{2}<0$ tais que $\tau^{n_{1}} x=\tau^{n_{2}} x=0$ mas $\tau^{n_{1}-1} x \neq 0$ e $\tau^{n_{2}+1} x \neq 0$. Obviamente, $\tau^{n_{1}-1} x$ é vértice projetivo e $\tau^{n_{2}+1}$ é vértice 
injetivo. Eles são únicos pois $\tau$ é bijetora.

Definição 1.4. Seja $\Delta$ dirigida e finita. A aljava-órbita $\mathcal{O}(\Delta)$ de $\Delta$ é definida da seguinte maneira: os vértice de $\mathcal{O}(\Delta)$ são as $\tau$-órbitas $o(x)$ dos vértices $x \in \Delta$, e existe uma flecha $o(x) \rightarrow o(y)$ se e somente se existir uma flecha $\tau^{-m} p_{x}=u \rightarrow p_{y}=$ $v$, para algum $m \geq 0 \mathrm{em} \Delta$ e o número de flechas de $o(x) \rightarrow o(y) \mathrm{em} \mathcal{O}(\Delta)$ é igual ao número de flechas de $u \rightarrow v$ em $\Delta$. Estamos denotando por $p_{x}$ e $p_{y}$ os únicos vértices projetivos na $\tau$-órbita de $x$ e na $\tau$-órbita de y respectivamente.

O conceito acima de aljava-órbita tem sido bastante útil no estudo das componentes conexas da aljava de Auslander-Reiten (ver seção 1.6).

Como estaremos também trabalhando com subaljavas de aljavas com translação, precisaremos introduzir aqui um outro conceito, o de grafo-órbita, que nos será mais útil para os resultados procurados.

Definição 1.5. Seja $\Delta^{\prime} \subseteq \Delta$ uma subaljava de $\Delta$. Definimos o grafo-órbita $\overline{\mathcal{O}}\left(\Delta^{\prime}\right)$ de $\Delta^{\prime}$ da seguinte maneira: os vértices de $\overline{\mathcal{O}}\left(\Delta^{\prime}\right)$ são as $\tau$-órbitas o $(x)$ em $\Delta^{\prime}$ dos vértices $x$ em $\Delta_{0}^{\prime}$ e existe uma aresta $o(x)-o(y)$ entre $o(x)$ e o $(y)$ em $\overline{\mathcal{O}}\left(\Delta^{\prime}\right)$ se existir flecha entre algum vértice $u$ representante de $o(x)$ e algum vértice $v$ representante de $o(y)$ em $\Delta^{\prime}$ e o número de arestas entre $o(x)$ e o(y) é igual ao número de flechas entre $u$ e $v$.

Consideremos uma aljava $\Delta$ dirigida, finita, com translação e sua aljava-órbita $\mathcal{O}(\Delta)$. Observe que o grafo subjacente de $\mathcal{O}(\Delta)$ coincide com o grafo-órbita $\overline{\mathcal{O}}(\Delta)$.

Afirmamos que se $\Delta^{\prime}$ é uma subaljava de $\Delta$, então $\overline{\mathcal{O}}\left(\Delta^{\prime}\right)$ é subgrafo de $\overline{\mathcal{O}}(\Delta)$. De fato, se $o(x)$ é um vértice em $\overline{\mathcal{O}}\left(\Delta^{\prime}\right)$ então existe $n \in \mathbb{Z} \backslash\{0\}$ tal que $\tau^{n} x \in \Delta_{0}^{\prime}$ e portanto $\tau^{n} x \in \Delta_{0}$. Desta forma $o(x)$ é um vértice de $\overline{\mathcal{O}}(\Delta)$.

Agora, se a aresta $o(x)-o(y)$ está em $\overline{\mathcal{O}}\left(\Delta^{\prime}\right)$, então existe uma flecha de um vértice em $o(x)$ para um vértice em $o(y)$ ou vice-versa em $\Delta^{\prime}$. Observamos que esta flecha também está em $\Delta$. Em ambos os casos se existir uma flecha $\tau^{s} x \rightarrow \tau^{t} y$ de um vértice em $o(x)$ para um vértice em $o(y)$ ou se existir uma flecha $\tau^{s^{\prime}} y \rightarrow \tau^{t^{\prime}} x$ em $\Delta^{\prime}$, concluímos que a aresta $o(x)-o(y)$ está em $\overline{\mathcal{O}}(\Delta)$. 
Exemplo 1.6. Consideremos a seguinte aljava dirigida com translação:

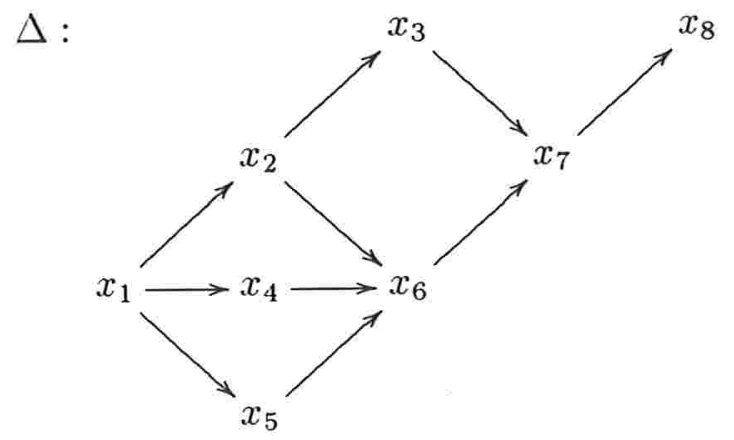

onde $x_{1}=\tau x_{6}, x_{2}=\tau x_{7}, x_{3}=\tau x_{8}$

Queremos construir a aljava-órbita de $\Delta$ :

$o\left(x_{1}\right)=\left\{x_{1}, x_{6}\right\}$

$o\left(x_{2}\right)=\left\{x_{2}, x_{7}\right\}$

$o\left(x_{3}\right)=\left\{x_{3}, x_{8}\right\}$

$o\left(x_{4}\right)=\left\{x_{4}\right\}, o\left(x_{5}\right)=\left\{x_{5}\right\}$.

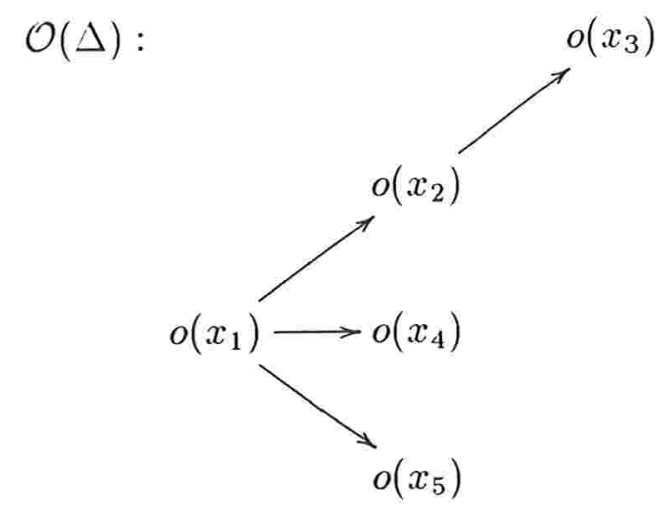

Observamos que $\mathcal{O}(\Delta)$ é uma aljava de tipo árvore. 
Finalmente, o grafo-órbita de $\Delta$ é o seguinte grafo:

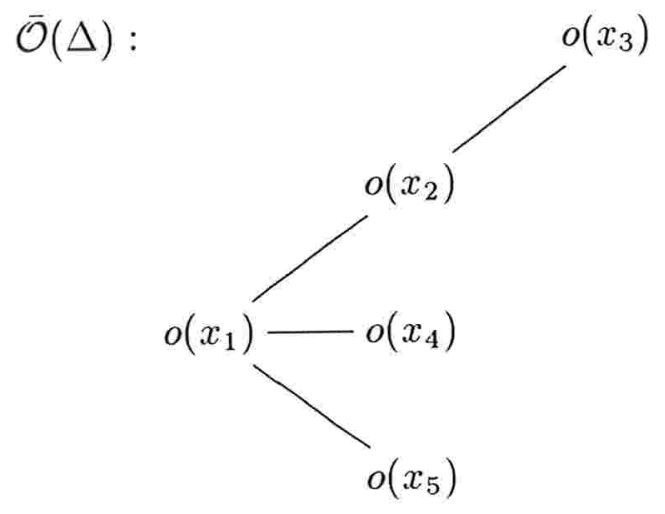

\section{4 Álgebras de caminhos}

Sejam $k$ um corpo e $\Delta=\left(\Delta_{0}, \Delta_{1}, s, e\right)$ uma aljava finita. Vamos definir a álgebra de caminhos $k \Delta$ a partir de $k$ e $\Delta$.

Seja inicialmente o conjunto $B$ formado por todos os caminhos de $\Delta$, incluindo os caminhos triviais. Consideramos agora $k \Delta$ o $k$-espaço vetorial com base $B$. Esta base é chamada base usual de $k \Delta$. Para que $k \Delta$ seja uma álgebra temos que definir uma multiplicação nos elementos da base $B$. Dados dois caminhos de $\Delta, \gamma_{1}=\alpha_{n} \ldots \alpha_{1}$ e $\gamma_{2}=\beta_{m} \ldots \beta_{1}$ definimos:

$$
\gamma_{1} \gamma_{2}= \begin{cases}\alpha_{n} \ldots \alpha_{1} \beta_{m} \ldots \beta_{1} & \text { se } e\left(\beta_{m}\right)=s\left(\alpha_{1}\right) \\ 0 & \text { caso contrário }\end{cases}
$$

Define-se então em $k \Delta$ uma estrutura de $k$-álgebra estendendo por linearidade a multiplicação acima a todos os seus elementos.

Seja $J_{\Delta}$ o ideal de $k \Delta$ gerado por todas as flechas de $\Delta$. Um ideal $I$ é admissível se existir um $n>0$ tal que $\left(J_{\Delta}\right)^{n} \subset I \subset\left(J_{\Delta}\right)^{2}$.

Observamos que se $\Delta$ é uma aljava finita e sem circuitos orientados, então todo ideal de $k \Delta$ contido em $\left(J_{\Delta}\right)^{2}$ é admissível.

Teorema 1.7. (Gabriel [28]) Seja $\Lambda$ uma álgebra indecomponível, básica e de dimensão finita sobre um corpo algebricamente fechado $k$. Então existe uma aljava 
$\Delta_{\Lambda}$ conexa e única salvo isomorfismos e um homomorfismo sobrejetor de álgebras $\phi: k \Delta_{\Lambda} \longrightarrow \Lambda$ tal que ker $\phi$ é um ideal admissível de $k \Delta$.

Dada uma álgebra $\Lambda$ básica, indecomponível e de dimensão finita, descreveremos a seguir como construir a sua aljava associada. Seja $\left\{e_{1}, \ldots, e_{n}\right\}$ um sistema completo de idempotentes ortogonais primitivos de $\Lambda$, numa ordem dada. (Tal sistema existe pois $\Lambda$ é de dimensão finita sobre $k$ ). Eles permitem decompor $\Lambda$ como soma de $\Lambda$-módulos indecomponíveis $P_{1} \oplus \cdots \oplus P_{n}$. Como $\Lambda$ é básica, $P_{i}$ não é isomorfo a $P_{j}$, para $i \neq j$. A aljava ordinária ou aljava associada a $\Lambda, \Delta_{\Lambda}$ é definida como tendo $n$ vértices $\left\{e_{1}, \ldots, e_{n}\right\}$. O número de flechas que começam em $e_{i}$ e terminam em $e_{j}$ será por definição

$$
\operatorname{dim}_{k} e_{j}\left(\frac{\operatorname{rad} \Lambda}{\operatorname{rad}^{2} \Lambda}\right) e_{i}
$$

Uma relação de um vértice $x$ para um vértice $y$ é uma combinação linear $\theta=\sum_{j=1}^{m} \lambda_{j} \omega_{j}$ onde, para cada $1 \leq j \leq m, \lambda_{j}$ é um escalar não nulo e $\omega_{j}$ um caminho de comprimento pelo menos dois de $x$ para $y$. A relação é chamada uma relação zero quando $m=1$ e uma relação de comutatividade quando $m=2 \mathrm{e}$ $\lambda_{1}=1$ e $\lambda_{2}=-1$.

Um conjunto $\rho$ contido em um ideal admissivel $I$ é um sistema de relações de $I$ se $\rho$ é um conjunto gerador de $I$ e todo elemento de $\rho$ é uma relação. É claro que todo ideal admissivel $I$ possui um sistema de relações.

Uma aljava com relações $(\Delta, \rho)$ é dada por uma aljava $\Delta$ e um sistema de relações $\rho$ de um ideal admissível $I$ de $k \Delta$. A álgebra de caminhos dada por uma aljava com relações $(\Delta, \rho)$ é, por definição a álgebra $\frac{k \Delta}{I}$. Então, para toda álgebra básica $\Lambda$ temos o isomorfismo $\Lambda \cong \frac{k \Delta_{\Lambda}}{I}$. Neste caso $\frac{k \Delta_{\Lambda}}{I}$ é denominada uma apresentação de $\Lambda$. Podemos reescrever o Teorema anterior da seguinte maneira:

Teorema 1.8. Toda álgebra de dimensão finita sobre um corpo algebricamente fechado $k$, básica e indecomponível, é uma álgebra de caminhos dada por uma aljava com relações. 
Exemplo 1.9. Seja $\Lambda$ uma $k$-álgebra dada pela aljava

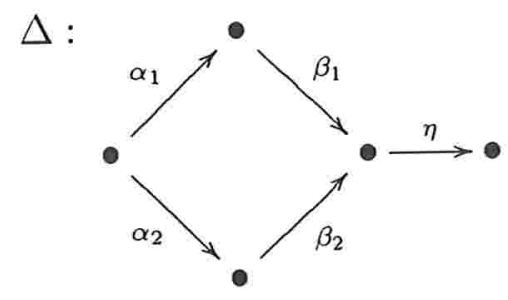

$\operatorname{com} \beta_{1} \alpha_{1}=\beta_{2} \alpha_{2}$ e $\eta \beta_{1}=0$.

Então a álgebra $\Lambda=\frac{k \Delta}{I}$ onde $\Delta$ é a aljava acima e $I$ é o ideal de $k \Delta$ gerado pelas relações $\beta_{1} \alpha_{1}-\beta_{2} \alpha_{2}$ e $\eta \beta_{1}$.

Recordemos que uma álgebra $\Lambda$ é hereditária se todo submódulo de um módulo projetivo é também um módulo projetivo. O próximo resultado caracteriza as álgebras com relações correspondentes às álgebras hereditárias.

Teorema 1.10. Uma álgebra $\Lambda=\frac{k \Delta}{I}$ é hereditária se e só se $I=0$ e $\Delta$ é dirigida.

Uma álgebra $\Lambda=\frac{k \Delta}{I}$ é denominada triangular se a sua aljava ordinária $\Delta$ é dirigida.

Observamos que uma álgebra $\Lambda=\frac{k \Delta}{I}$ pode equivalentemente ser considerada como uma $k$-categoria cuja classe de objetos é o conjunto $\Delta_{0}$, e o conjunto de morfismos de $x$ para $y$ é o espaço vetorial $k \Delta(x, y)$ de todas as combinações lineares de caminhos em $\Delta$ de $x$ para $y$ módulo o subspaço $I(x, y)=I \cap k \Delta(x, y)$. (Para mais detalhes ver [16]).

\subsection{Módulos e representações de álgebras}

Já vimos como as álgebras podem ser descritas em termos de aljavas com relações. Agora, queremos visualizar as representações de uma álgebra, quer dizer, os seus módulos através das representações da correspondente aljava com relações. 
Seja $(\Delta, \rho)$ uma aljava com relações. Uma representação de $\Delta$ é dada por $V=\left(\left(V_{i}\right)_{i \in \Delta_{0}},\left(f_{\alpha}\right)_{\alpha \in \Delta_{1}}\right)$ onde para cada $i \in \Delta_{0}, V_{i}$ é um espaço vetorial de dimensão finita sobre $k$ e para cada $\alpha \in \Delta_{1}, f_{\alpha}$ é uma transformação linear. Dado um caminho não trivial $\gamma=\alpha_{n} \cdots \alpha_{1}$ de $i$ a $j$ podemos definir $V(\gamma)$ como sendo a transformação linear de $V_{i}$ a $V_{j}$ dada pela composta $f_{\alpha_{n}} \cdots f_{\alpha_{1}}$. Podemos estender esta definição para uma combinação linear de caminhos por linearidade.

Definição 1.11. a) Dizemos que a representação $V$ satisfaz uma relação $r$ se $V(r)=$ 0 e que satisfaz o sistema de relações de $\rho$ se satisfizer cada relação $r$ de $\rho$.

b) Uma representação de $(\Delta, \rho)$ é uma representação de $\Delta$ que satisfaz as relações $\rho$.

Dadas duas representações $V=\left(\left(V_{i}\right)_{i \in \Delta_{0}},\left(f_{\alpha}\right)_{\alpha \in \Delta_{1}}\right)$ e $W=\left(\left(W_{i}\right)_{i \in \Delta_{0}},\left(g_{\alpha}\right)_{\alpha \in \Delta_{1}}\right)$ um morfismo $\phi: V \rightarrow W$ é dado por uma família de transformações lineares $\phi=\left(\phi_{i}\right)_{i \in \Delta_{0}}$ tal que para cada flecha $\alpha: i \rightarrow j$ o seguinte diagrama comuta:

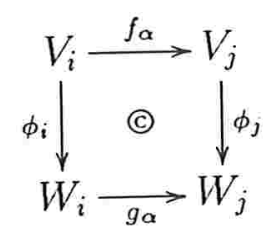

isto é, $\phi_{j} f_{\alpha}=g_{\alpha} \phi_{i}$.

A composição dos morfismos é definida naturalmente coordenada a coordenada. Com isto, temos definida uma categoria chamada a categoria das representações de $(\Delta, \rho)$ e a denotaremos por $\bmod (\Delta, \rho)$. Considere agora $\Lambda$ uma $k$-álgebra de dimensão finita sobre um corpo algebricamente fechado $k$, indecomponível e básica. Sabemos do Teorema 1.5 que $\Lambda$ é uma álgebra de caminhos dada por uma aljava com relações $(\Delta, \rho)$, isto é $\Lambda \cong \frac{k \Delta}{I}$ onde $\rho$ é o sistema de relações do ideal admissível $I$.

Teorema 1.12. Com as notaçôes acima $\bmod (\Delta, \rho)$ e $\bmod \Lambda$ são equivalentes como $k$-categorias.

Para mais detalhes a respeito desta seção recomendamos ver [22] e [20]. 


\subsection{Sequências e aljavas de Auslander-Reiten}

Iremos nesta seção descrever resultados que surgiram nos anos 70 e que influenciaram definitivamente o rumo da teoria desde então. M. Auslander e I. Reiten introduziram a noção de sequências quase cindidas (atualmente chamadas de sequências de Auslander-Reiten).

Apresentaremos aqui as definições básicas e alguns resultados importantes. Para uma leitura mais detalhada ver [11], [13], [22] e [40].

Definição 1.13. Uma sequência exata curta de $\Lambda$-módulos que não cinde

$$
0 \longrightarrow N \stackrel{f}{\longrightarrow} E \stackrel{g}{\longrightarrow} M \longrightarrow 0
$$

é uma sequência de Auslander-Reiten se:

i) os seus extremos $N$ e $M$ são indecomponíveis;

ii) para todo morfismo $h: X \rightarrow M, X \in \bmod \Lambda$, que não seja um epimorfismo que cinde (isto é, que não tenha secção), existe $\bar{h}: X \rightarrow E$ tal que g $\bar{h}=h$;

ii') para todo $h: N \rightarrow X, X \in \bmod \Lambda$, que não seja um monomorfismo que cinde (isto é, que não tenha retração), existe $\bar{h} f=h$.

Observamos que as condições ii) e ii') são equivalentes.

Veremos a seguir em que casos existem sequências de Auslander-Reiten e que nestes casos ela é única, salvo isomorfismos.

Teorema 1.14. (Existência e Unicidade): Sejam $\Lambda$ uma álgebra e $M$ um $\Lambda$-módulo indecomponivel.

1. Se $M$ não for projetivo então existe e é única, salvo isomorfismos, a sequência de Auslander-Reiten terminando em $M: 0 \rightarrow N \rightarrow E \rightarrow M \rightarrow 0$.

2. Se $M$ não for injetivo então existe e é única, salvo isomorfismos, a sequência de Auslander-Reiten começando em $M: 0 \rightarrow M \rightarrow E \rightarrow N \rightarrow 0$ 
Vamos estudar os morfismos que compõem as sequências de Auslander-Reiten a partir da propriedade de levantamento ii).

Definição 1.15. Seja $M$ um módulo indecomponível.

1. Um morfismo $g: E \rightarrow M$ é um poço em $M$ se:

(i) $g$ não for um epimorfismo que cinde (isto é, $g$ não tem secção).

(ii) para todo $h: E \rightarrow E$ tal que $g h=g$, $h$ é um automorfismo.

(iii) para todo $h: X \rightarrow M$ que não seja um epimorfismo que cinde, existe $h^{\prime}: X \rightarrow$ E tal que $g h^{\prime}=h$.

2. Um morfismo $f: M \rightarrow E$ é uma fonte em $M$ se:

(i) $f$ não for um monomorfismo que cinde (isto é, $f$ não tem retração).

(ii) para todo $h: E \rightarrow E$ tal que $h f=f, h$ é um automorfismo.

(iii) para todo $h: M \rightarrow X$ que não seja um monomorfismo que cinde, existe $h^{\prime}: E \rightarrow E$ tal que $h^{\prime} f=h$.

Proposição 1.16. Seja $0 \longrightarrow N \stackrel{f}{\longrightarrow} E \stackrel{g}{\longrightarrow} M \longrightarrow 0$ uma sequência de AuslanderReiten. Então

(a) $f$ é uma fonte e qualquer outra fonte em $N$ é isomorfa a $f$.

(b) $g$ é um poço e qualquer outro poço em $M$ é isomorfo a $g$.

Quando o módulo for projetivo ou injetivo, vejamos na seguinte Proposição o que ocorre com os morfismos fonte e poço.

Proposição 1.17. 1. Se $P$ é um projetivo indecomponível não simples então a inclusão $i: \operatorname{radP} \hookrightarrow P$ é um poço e todo outro poço em $P$ é isomorfo a $i$.

2. Se I é um injetivo indecomponível não simples então a projeção natural $\pi$ : $I \rightarrow \frac{I}{s o c I}$ é uma fonte e toda outra fonte em I é isomorfa a $\pi$.

Veremos que os morfismos das sequências de Auslander-Reiten possuem certas propriedades de irredutibilidade. 
Definição 1.18. Um morfismo que não cinde $f \in \operatorname{Hom}_{\Lambda}(X, Y) \operatorname{com} X, Y$ em $\bmod \Lambda$ é irredutivel se para cada decomposição $f=g h$ ou $g$ é um epimorfismo que cinde ou $h$ é um monomorfismo que cinde.

Observamos que se $f: X \rightarrow Y$ é irredutível então $f$ é um epimorfismo ou um monomorfismo. Relacionaremos a seguir os morfismos irredutíveis com fontes e poços.

Proposição 1.19. Seja $M$ um $\Lambda$-módulo indecomponível.

(a) Um morfismo $h: M \rightarrow X$ é irredutivel se e só se existe $h^{\prime}: M \rightarrow X^{\prime}$ tal que $\left(h, h^{\prime}\right)$ é uma fonte em $M$.

(b) Um morfismo $h: X \rightarrow M$ é irredutivel se e só se existe $h^{\prime}: X^{\prime} \rightarrow M$ tal que $\left(h, h^{\prime}\right)$ é um poço em $M$.

Vamos definir os subgrupos $\operatorname{rad}^{n}(X, Y)$ de $\operatorname{Hom}_{\Lambda}(X, Y)$ para $X$ e $Y$ em ind $\Lambda$ e $n \geq 1$. Isto é feito indutivamente como segue.

(i) Para $n=1 \operatorname{rad}^{1}(X, Y)=\operatorname{rad}(X, Y)$ é o subgrupo dos morfismos de $X$ em $Y$ que não cindem.

(ii) Suponhamos que $\operatorname{rad}^{i}(X, Y)$ está definido para $1 \leq i \leq n$, então $f$ está em $\operatorname{rad}^{n}(X, Z)$ se e só se pode escrever-se como soma de morfismos da forma $g h$ com $h \in \operatorname{rad}^{n}(X, Z)$ e $g \in \operatorname{rad}^{n^{\prime \prime}}(Z, Y)$ para algum $Z$ em ind $\Lambda$, e com $n^{\prime}$ e $n^{\prime \prime}$ menores que $n$ mas satisfazendo $n^{\prime}+n^{\prime \prime} \geq n$.

(iii) $\operatorname{rad}^{\infty}(X, Y)$ se define como a intersecção dos $\operatorname{rad}^{n}(X, Y), n \in \mathbb{N}$.

Proposição 1.20. Seja $f: Y \rightarrow X$ um morfismo entre módulos em ind $Y$ que não seja um isomorfismo. Então $f=\sum \alpha_{i}+\beta$, onde cada $\alpha_{i}: Y \rightarrow X$ é uma composta finita de morfismos irredutiveis entre módulos indecomponíveis e $\beta$ está em $\operatorname{rad}^{\infty}(Y, X)$.

Iremos a seguir associar a uma dada álgebra $\Lambda$ uma aljava $\Gamma_{\Lambda}$ onde os vértices e as flechas representarão respectivamente os $\Lambda$-módulos indecomponíveis e os mor- 
fismos irredutíveis entre eles. Tal aljava $\Gamma_{\Lambda}$ é chamada de aljava de AuslanderReiten de $\Lambda$ e foi formalizada por C.M. Ringel. Ver, por exemplo, [40].

Seja $M$ um $\Lambda$-módulo indecomponível. Quando $M$ não é projetivo sabemos que existe uma sequência de Auslander-Reiten terminando em $M$ :

$$
0 \longrightarrow N \longrightarrow E \longrightarrow M \longrightarrow 0
$$

Lembramos que o $\Lambda$-módulo $N$ é indecomponível e $N \cong \operatorname{DTr} M$ que é o dual da transposta de $M$ definido em [10]. Devido à unicidade da sequência de AuslanderReiten temos que $D \operatorname{Tr} M$ é único a menos de isomorfismos. O módulo $\operatorname{Tr} M$ é dito o transladado de Auslander-Reiten de $M$.

Por outro lado, se $M$ não for injetivo então existe uma única sequência de Auslander-Reiten começando em $M$ :

$$
0 \longrightarrow M \longrightarrow E \longrightarrow N \longrightarrow 0
$$

e $N$ é um $\Lambda$-módulo indecomponível e $N \cong \operatorname{Tr} \mathrm{D} M$ e será dito o transladado inverso de Auslander-Reiten de $M$.

Definição 1.21. Seja $\Lambda$ uma álgebra. A aljava de Auslander-Reiten de $\Lambda$ é a aljava definida como segue:

1. Os vértices estão em correspondência biunívoca com as classes de isomorfismos dos $\Lambda$-módulos indecomponíveis, isto é, para cada indecomponível $M$ associamos um vértice $[M]$ e os vértices $[M]$ e $\left[M^{\prime}\right]$ são os mesmos se e só se $M \cong M^{\prime}$.

2. O número de flechas de $[M] \rightarrow\left[M^{\prime}\right]$ é dado por $\operatorname{dim}_{k} \operatorname{Irr}\left(M, M^{\prime}\right)$, onde

$$
\operatorname{Irr}\left(M, M^{\prime}\right)=\frac{\operatorname{rad}\left(M, M^{\prime}\right)}{\operatorname{rad}^{2}\left(M, M^{\prime}\right)}
$$

é o conjunto dos morfismos irredutiveis de $M$ em $M^{\prime}$.

Algumas propriedades da aljava $\Gamma_{\Lambda}$ : 
1. A aljava $\Gamma_{\Lambda}$ não tem laços.

2. $\Gamma_{\Lambda}$ é uma aljava localmente finita.

3. Dado $M \in i n d \Lambda$ não projetivo então o número de flechas que saem de [Dtr $M$ ] é igual ao número de flechas que chegam em $[M]$.

Observamos que a aljava de Auslander-Reiten $\Gamma_{\Lambda}$ é uma aljava com translação pois é localmente finita, não tem laços e a aplicação $\tau$ bijetora é dada por DTr $M$ que é o transladado de Auslander-Reiten como definido acima.

Proposição 1.22. Seja $\Lambda$ uma álgebra indecomponível de tipo de representção finito. Então a aljava de Auslander-Reiten de $\Lambda$ é conexa.

Quando a aljava de Auslander-Reiten $\Gamma_{\Lambda}$ não possui circuitos orientados dizemos que a álgebra $\Lambda$ é de representação direta.

Observamos que para toda subaljava $\Delta$ finita, dirigida da aljava de AuslanderReiten $\Gamma_{\Lambda}$ de uma álgebra $\Lambda$ podemos definir a aljava-órbita $\mathcal{O}(\Delta)$ e seu respectivo grafo-órbita $\overline{\mathcal{O}}(\Delta)$ definidos na seção 1.2 .

Uma álgebra $\Lambda$ é dita de tipo de representação finito ou de representação finita se o número de classes de isomorfia de $\Lambda$-módulos indecomponíveis é finito. Caso contrário, é dita de tipo de representação infinito ou de representação infinita.

\subsection{Componentes pós-projetivas}

Seja $\Lambda$ uma $k$-álgebra de dimensão finita. Seja $\Gamma_{\Lambda}$ a aljava de Auslander-Reiten de $\Lambda$.

Definição 1.23. Uma componente conexa $\mathcal{P}$ de $\Gamma_{\Lambda}$ é chamada uma componente pós-projetiva quando $\mathcal{P}$ não contiver circuitos orientados e cada $X \in \mathcal{P}$ for da forma $(\operatorname{Tr} D)^{r} P$ para algum $r \in \mathbb{N}$ e $P$ um $\Lambda$-módulo projetivo indecomponível. 
Um dos fatos importantes de um módulo $X$ estar em uma componente pósprojetiva é que $X$ é homologicamente trivial, isto é, $\operatorname{Ext}_{\Lambda}^{i}(X, X)=0$ para $i>0$, $E n d_{\Lambda} X=k$ e sua classe de isomorfismo é unicamente determinada por seus fatores de composição.

Muitas classes de álgebras tem componentes pós-projetivas, como as álgebras hereditárias ( [29], [40]) e as álgebras inclinadas ( [44]), álgebras satisfazendo a condição de separação ( [15], [17]) e as álgebras quase inclinadas ( [23]).

Em [26], Dräxler e de la Peña deram um procedimento algorítmico para decidir a existência de componentes pós-projetivas. Antes de enuncia-lo precisaremos de alguns conceitos ainda não apresentados neste trabalho.

Dada uma aljava finita e dirigida, $\Delta$, definimos a subaljava $\Delta^{x}$ de $\Delta$ como a aljava obtida quando retiramos todos os vértices $y$ de $\Delta$ tal que exista caminho de $x$ para $y$ (incluindo o caminho trivial de $x$ em $x$ ).

Definiremos agora os módulos dirigidos que têm sido importantes na teoria de representações de álgebra pois componentes pós-projetivas e componentes pré- injetivas em geral e componentes conexas de álgebras inclinadas consistem inteiramente de módulos dirigidos. Para módulos indecomponíveis temos a seguinte definição:

Definição 1.24. Um módulo indecomponível $M$ de $\bmod \Lambda$ é chamado módulo dirigido se ele pertencer a um ciclo $M \rightarrow M_{1} \rightarrow M_{2} \rightarrow \ldots \rightarrow M_{n} \rightarrow M$ de não isomorfismos não nulos indecomponíveis de $\bmod \Lambda$.

Happel e Ringel mostram que um módulo indecomponível $M$ ser dirigido no sentido acima é equivalente a não existir um módulo não projetivo indecomponível $W$ tal que existem caminhos de $X$ para $W$ e de $W$ para $X$. Com esta caracterização eles estenderam a definição de módulos dirigidos indecomponíveis para módulos arbitrários (não necessariamente indecomponíveis), vejamos abaixo.

Definição 1.25. [39]: Um módulo $M$ será chamado módulo dirigido quando não existirem somandos diretos indecomponiveis $M_{1}, M_{2}$ de $M$, e um módulo não projetivo 
indecomponivel $W$ tal que existem um caminho de $M_{1}$ para $\tau W$ e um caminho de $W$ para $M_{2}$.

Teorema 1.26. ([26]): Seja $\Lambda=\frac{k \Delta}{I}$ uma $k$-álgebra de dimensão finita tal que $\Delta$ é dirigida. Então $\Gamma_{\Lambda}$ tem uma componente pós-projetiva se e somente se para cada vértice $x \in \Delta_{0}$ uma das seguintes propriedades é satisfeita:

$(\mathbf{1 x})$ existe uma componente pós-projetiva $\mathcal{P}$ de $\Gamma_{\Lambda^{x}}\left(\Lambda^{x}=\frac{k \Delta^{x}}{I}\right)$ tal que os somandos do $\operatorname{rad} P_{x}(\Lambda)$ não estão em $\mathcal{P}$;

(2x) o conjunto de predecessores de cada somando do $\operatorname{rad}_{x}(\Lambda)$ em $\bmod _{\Lambda^{x}}$ é finito e formado por módulos dirigidos. Mais ainda, se $x$ é um poço, então $\operatorname{rad} P_{\Lambda}(x)$ é dirigido em $\bmod _{\Lambda^{x}}$.

\subsection{Extensão por um ponto de álgebras}

Seja $B$ uma álgebra e $M$ um $B$-módulo. A extensão por um ponto de $B$ por $M$ é por definição a $k$-álgebra de dimensão finita:

$$
B[M]=\left(\begin{array}{cc}
B & 0 \\
M & k
\end{array}\right)
$$

com as operações usuais de matrizes.

Um módulo em $B[M]$ é identificado com a tripla $(X, U, \varphi)$ onde $X \in \bmod B$ e $U \in \operatorname{modk}$ e $\varphi: U \longrightarrow \operatorname{Hom}(M, X)$ é um morfismo $k$-linear. Temos:

$$
\left(\begin{array}{l}
X_{1} \\
X_{2}
\end{array}\right) \quad\left(\begin{array}{cc}
B & 0 \\
M & k
\end{array}\right)=\left(\begin{array}{c}
X_{1} B+X_{2} M \\
X_{2} k
\end{array}\right)
$$

onde $X_{2} . M$ é definida por $\tilde{\varphi}: X_{2} \otimes_{k} M \longrightarrow X_{1}$, mas como $\operatorname{Hom}_{k}\left(X_{2}, \operatorname{Hom}_{B}\left(X_{2}, X_{1}\right)\right) \cong$ $\operatorname{Hom}_{B}\left(X_{2} \otimes_{k} M, X_{1}\right)$ usamos então $\varphi$ que é adjunta de $\tilde{\varphi}$.

Da definição da extensão por um ponto nós vemos que uma condição necessária para uma álgebra $\Lambda$ ser da forma $B[M]$ para alguma álgebra $B$ e um $B$-módulo $M$ é 
que exista um $\Lambda$-módulo simples injetivo.

Por outro lado, suponhamos que uma álgebra $\Lambda$ admita um módulo injetivo simples $S$. Seja $P(S)$ uma cobertura projetiva de $S$ e seja $e \in \Lambda$ um idempotente tal que $P(S)=e \Lambda$. Seja $B=\frac{\Lambda}{\langle e\rangle}$ (onde $\langle e\rangle$ denota o ideal bilateral em $\Lambda$ gerado pelo idempotente e seja $M=\operatorname{rad} P(S)$. Então $M$ é um $B$-módulo e não é difícil verificar que $\Lambda \simeq B[M]$. Vejamos o seguinte exemplo:

Exemplo 1.27. Consideremos a álgebra $\Lambda=\frac{k \Delta}{\rho}$

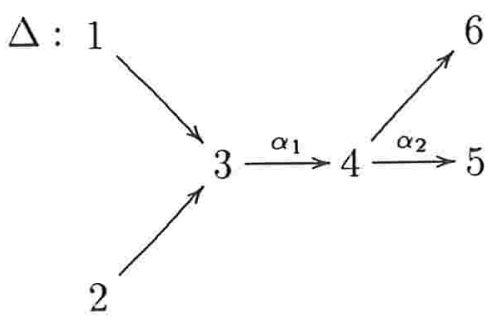

onde $\rho$ é gerado por $\alpha_{2} \alpha_{1}$.

Como $I_{6}$ é um $\Lambda$-módulo injetivo simples, temos $M=\operatorname{rad}_{6}$ e $B=\frac{\Delta^{\prime}}{\rho}$ onde

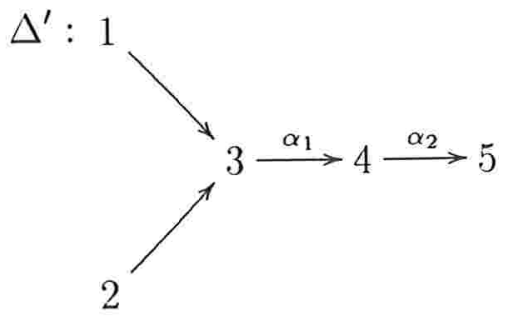

e $\rho$ é gerado por $\alpha_{2} \alpha_{1}$. Então podemos ver a álgebra $\Lambda$ como $\Lambda=B[M]$

Existe um conceito dual chamado coextensão por um ponto. Denotamos $M^{*}$ o $k$-dual de $M$. Então a coextensão por um ponto $[M] B$ de $B$ por $M$ é por definição a $k$-álgebra de dimensão finita:

$$
[M] B=\left(\begin{array}{cc}
B & M^{*} \\
0 & k
\end{array}\right)
$$

com as operações usuais de matrizes. 


\subsection{Cohomologia de Hochschild}

Para um bimódulo finitamente gerado $X$, os grupos de Cohomologia de Hochschild $H^{i}(\Lambda, X), i>0$ foram introduzidos em [35]. Os grupos de Cohomologia de Hochschild com dimensão baixa, $i \leq 2$ possuem uma interpretação bastante concreta de estruturas de álgebras clássicas tais como derivações e extensões. Estes grupos têm representado um importante papel na teoria de representações de álgebras: $H^{1}(\Lambda)$ está relacionado com as propriedades de separação dos vértices da aljava de $\Lambda$ (ver [2], [32], [42]); o anulamento de $H^{2}(\Lambda)$ está relacionado com a existência de bases multiplicativas para $\Lambda([37])$.

Sejam $k$ um corpo algebricamente fechado e $\Lambda$ uma $k$-álgebra de dimensão finita conexa e básica. Seja $X$ um $\Lambda$-bimódulo que é de dimensão finita sobre $k$. Nós definimos o complexo de Hochschild $C^{\bullet}$ como segue:

$C_{i}=0, d^{i}=0$ para $i<0, C^{\circ}=X, C^{i}=H o m_{k}\left(\Lambda^{\otimes i}, X\right)$ para $i>0\left(\Lambda^{\otimes i}=\Lambda \otimes_{k} \ldots \otimes_{k} \Lambda\right.$ onde $\Lambda$ aparece $i$ vezes $), d^{0}: X \rightarrow \operatorname{Hom}_{k}(\Lambda, X) \operatorname{com}\left(d^{0} x\right)(a)=a x-x a$ para $x \in X$ e $a \in \Lambda, d^{i}: C^{i} \rightarrow C^{i+1} \mathrm{com}$

$$
\begin{gathered}
\left(d^{i} f\right)\left(a_{1} \otimes \cdots \otimes a_{i+1}\right)=a_{1} f\left(a_{2} \otimes \cdots \otimes a_{i+1}\right)+ \\
+\sum_{j=1}^{i}(-1)^{j} f\left(a_{1} \otimes \cdots \otimes a_{j} a_{j+1} \otimes \cdots \otimes a_{i+1}\right)+ \\
+(-1)^{i+1} f\left(a_{1} \otimes \cdots \otimes a_{i}\right) a_{i+1}
\end{gathered}
$$

para $f \in C^{i}$ e $a_{1}, \ldots, a_{i+1} \in \Lambda$.

Nós definimos $H^{i}(\Lambda, X)=H^{i}\left(C^{\bullet}\right)=\frac{k e r d^{i}}{i m d^{i-1}}$ como o $i$-ésimo grupo de cohomologia de $\Lambda$ com coeficientes no bimódulo $X$. Nosso interesse em particular é o caso em que $X$ é igual a álgebra $\Lambda$ vista como bimódulo sobre si mesma. Neste caso denotamos $H^{i}(\Lambda, \Lambda)$ simplesmente por $H^{i}(\Lambda)$.

Vamos estudar os grupos de Cohomologia de dimensão baixa. $H^{0}(\Lambda, X)=X^{\Lambda}=\{x \in X / a x=x a \forall a \in \Lambda\}$. Observamos que $H^{0}(\Lambda)$ coincide com o centro de $\Lambda$. 
Definição 1.28. (1) Uma derivação de $\Lambda$ em $X$ é um morfismo $\delta \in \operatorname{Hom}_{k}(\Lambda, X)$ satisfazendo a propriedade $\delta(a b)=a \delta(b)+\delta(a) b$ para todos $a, b \in \Lambda$. Ok-espaço das derivações de $\Lambda$ em $X$ é denotado $\operatorname{por} \operatorname{Der}(\Lambda, X)$.

(2) Uma derivação interna é um morfismo $\delta_{x}: \Lambda \rightarrow X$ tais que $\delta_{x}(a)=a x-x a$ com $x \in X$. O subspaço das derivações internas de $\Lambda$ em $X$ é denotado por $\operatorname{Der}^{0}(\Lambda, X)$.

Temos então da definição (ver [35]) que

$$
H^{1}(\Lambda, X)=\frac{\operatorname{Der}(\Lambda, X)}{\operatorname{Der}^{0}(\Lambda, X)}
$$

Além da descrição acima temos uma descrição mais fácil de ser usada que foi obtida por Happel em [32].

Seja $\left\{e_{1}, \ldots, e_{n}\right\}$ um sistema completo de idempotentes ortogonais primitivos de $\Lambda$. Seja $\operatorname{Der}^{n}(\Lambda)=\left\{\delta \in \operatorname{Der}(\Lambda) / \delta\left(e_{i}\right)=0\right.$ para $\left.1 \leq i \leq n\right\}$ o subspaço das derivações normalizadas. Por $\operatorname{Der}^{n, 0}(\Lambda)$ nós denotamos o subspaço $\operatorname{Der}^{n}(\Lambda) \cap \operatorname{Der}^{0}(\Lambda)$. Então

$$
H^{1}(\Lambda)=\frac{\operatorname{Der}^{n}(\Lambda)}{\operatorname{Der}^{n, 0}(\Lambda)} .
$$

Para $k$-álgebras hereditárias de dimensão finita, Happel provou em [32]:

Proposição 1.29. Seja $\Delta$ uma aljava finita e dirigida. Então $H^{1}(k \Delta)=0$ se e somente se $\Delta$ é uma aljava de tipo árvore.

Para $\Lambda=\frac{k \Delta}{I}$ com $\Delta$ aljava finita e dirigida segue de [32] que se $\Lambda$ é de representação direta então $H^{i}(\Lambda)=0$ para $i \geq 2$. Nas próximas seções veremos que para uma álgebra $\Lambda$ de representação direta e simplesmente conexa, o seu primeiro grupo de Cohomologia de Hochschild se anula ( [32]) e alguns cálculos dos grupos de Cohomologia de Hochschild para as álgebras de Auslander ( [33]). 


\subsection{0 Álgebras simplesmente conexas}

Seja $(\Delta, I)$ uma aljava conexa e com relações. Uma relação $\rho=\sum_{j=1}^{m} \lambda_{j} \omega_{j} \in I(x, y)$ é dita ser minimal se $m \geq 2$ e para todo subconjunto próprio não vazio $J$ de $\{1, \ldots, m\}, \sum_{j \in J} \lambda_{j} \omega_{j} \notin I$. Denotamos por $m(I)$ o conjunto das relações minimais do ideal $I$.

No conjunto dos passeios em $\Delta$ nós definimos a relação de homotopia $\sim$ (ver [36]) como a menor relação de equivalência satisfazendo as seguintes condições:

(a) $\alpha \alpha^{-1} \sim w_{x} \sim \beta^{-1} \beta$ para cada flecha $\alpha \in \Delta_{1}$ com poço $x$ e cada flecha $\beta \in \Delta_{1}$ com fonte $x$ onde $w_{x}$ é o caminho trivial de $x$ para $x$.

(b) $u \sim v$ para quaisquer dois caminhos $u, v$ com mesma fonte e mesmo poço e tal que exista $\sum_{j=1}^{m} \lambda_{j} \gamma_{j} \in m(I)$ com $u=\gamma_{i}, v=\gamma_{j}$ para alguns $i, j$.

(c) A relação $u \sim v$ implica $w u \sim w v$ e $u p \sim v p$ sempre que isto fizer sentido.

Obviamente a composição de passeios induz um produto de classes de homotopia: se $\bar{w}$ denota a classe de homotopia de um passeio $w$, então o produto $\bar{u} \bar{v}$ está definido sempre que $u v$ está definido, e nós temos $\bar{u} \bar{v}=\overline{u v}$. Em particular, para cada $x_{0} \in \Delta_{0}$, a composição está em todo lugar definida no conjunto $\pi_{1}\left(\Delta, I, x_{0}\right)$ das classes de homotopia dos passeios de $x_{0}$ para $x_{0}$. Então $\pi_{1}\left(\Delta, I, x_{0}\right)$ é um grupo; o grupo fundamental de $(\Delta, I)$ em $x_{0}$. Como $\Delta$ é uma aljava conexa, para quaisquer dois $x, y \in$ $\Delta_{0}$, os grupos $\pi_{1}(\Delta, I, x)$ e $\pi_{1}(\Delta, I, y)$ são obviamente isomorfos. Nós denotamos por $\pi_{1}(\Delta, I)$ o grupo $\pi_{1}(\Delta, I, x)$ em um vértice fixado $x$ de $\Delta$ e o chamamos de grupo fundamental de $(\Delta, I)$.

Definição 1.30. [3] Uma álgebra triangular $\Lambda$ é chamada simplesmente conexa se, para toda apresentação $\Lambda \simeq \frac{k \Delta}{I}$ de $\Lambda$, o grupo fundamental $\pi_{1}(\Delta, I)$ é trivial.

Para uma álgebra $\Lambda$ de tipo de representação finito, uma estratégia conhecida para descrever a categoria $\bmod \Lambda$ é usar técnicas de recobrimento porque sabemos que 
podemos levantar os módulos de $\bmod \Lambda$ a módulos indecomponíveis de uma álgebra simplesmente conexa (ver [18]). E assim reduzimos o problema pois vamos estudar uma álgebra simplesmente conexa de tipo de representação finito que é uma classe de álgebra bem conhecida.

Segue de [18] e [36] que a definição 1.30 coincide com a definição introduzida em [16] para álgebras de tipo de representação finito.

Teorema 1.31. Uma álgebra triangular $\Lambda$ é simplesmente conexa se e somente se não admite nenhum recobrimento de Galois próprio.(Ver [41]).

Teorema 1.32. [16] Uma k-álgebra de dimensão finita básica, conexa de tipo de representação finito é simplesmente conexa se e somente se sua aljava de AuslanderReiten não possui circuito orientado e seu grafo-órbita é uma árvore orientada.

Em [15](ver também [14]) foi introduzida a condição de separação que definiremos a seguir:

Seja $\Lambda=\frac{k \Delta}{I}$ uma álgebra triangular. Denominamos um vértice $x$ de $\Delta$ de separante se o radical de $P_{x}(\Lambda)$ é uma soma direta de módulos indecomponíveis não isomorfos dois a dois cujos suportes estão contidos em diferentes componentes conexas da subaljava $\Delta^{x}$ de $\Delta$. Se todos os vértices de $\Delta$ forem separantes, diremos que $\Lambda$ tem a condição de separação.

Em [15], foi obtido o seguinte critério para álgebras de tipo de representação finito que decide em termos da aljava ordinária com relações de $\Lambda$ quando a álgebra $\Lambda$ é simplesmente conexa ou não:

Teorema 1.33. $\Lambda$ é simplesmente conexa se e somente se satisfaz a condição de separação. 
Exemplo 1.34. Considere a álgebra $\Lambda=\frac{k \Delta}{I}$ onde

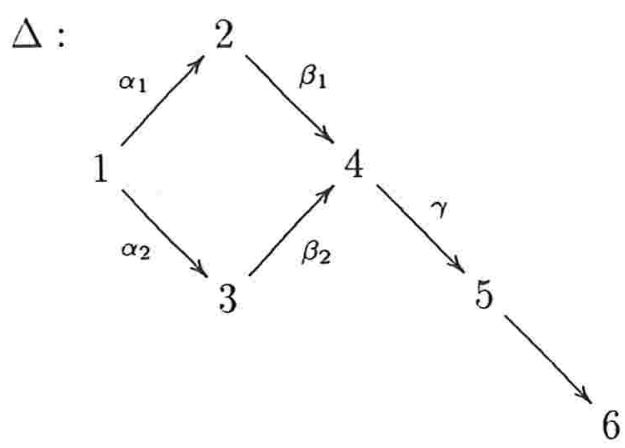

$\operatorname{com} I=<\beta_{1} \alpha_{1}-\beta_{2} \alpha_{2}, \gamma \beta_{2}>$.

Observamos que $\Lambda$ é uma álgebra de tipo de representação finito. Portanto, como todos os vértices de $\Delta$ são separantes, podemos afirmar pelo teorema acima que $\Lambda$ é simplesmente conexa.

Em [32], Happel mostrou que para uma álgebra $\Lambda$ de tipo de representação finito temos:

Teorema 1.35. Seja $\Lambda$ de representação direta. Então $\Lambda$ é simplesmente conexa se e somente se $H^{1}(\Lambda)=0$.

Outros resultados foram obtidos para álgebras simplesmente conexas de tipo de representação finito em [16] e [18] por exemplo. Pouco se sabe sobre técnicas de recobrimento para álgebras de tipo de representação infinito, mas algumas álgebras simplesmente conexa de tipo de representação infinito têm sido descritas (ver [5], [4], [25], [38]). Alguns exemplos de álgebras simplesmente conexas de tipo de representação infinito são as álgebras inclinadas de tipo de representação infinito de tipos Euclidianos $\tilde{\mathbf{D}}_{n}, \tilde{\mathbf{E}}_{p}(n \geq 4, p=6,7,8)$ e as álgebras tubulares de Ringel.

É interessante calcularmos as cohomologias de Hochschild de álgebras simplesmente conexas, e investigar a relação existente entre o anulamento de $H^{1}(\Lambda)$ e o fato de $\Lambda$ ser simplesmente conexa. (Olhar [32]).

Em [42] temos o seguinte resultado: 
Proposição 1.36. Toda álgebra triangular satisfazendo a condição de separação é simplesmente conexa.

Definição 1.37. Uma álgebra $\Lambda$ é dita fortemente simplesmente conexa se toda subcategoria plena convexa de $\Lambda$ for simplesmente conexa.

Obviamente temos que se $\Lambda$ é fortemente simplesmente conexa então $\Lambda$ é simplesmente conexa.

Observamos que toda álgebra cuja aljava ordinária é do tipo árvore é fortemente simplesmente conexa.

Para o caso em que $\Lambda$ é de representação finita, temos que $\Lambda$ é fortemente simplesmente conexa se e somente se satisfaz a condição de separação se e somente se é simplesmente conexa.

O principal resultado em [42] foi a seguinte caracterização da classe das álgebras fortemente simplesmente conexas:

Teorema 1.38. Seja $\Lambda$ uma álgebra triangular. Então as seguintes condições são equivalentes:

(i) $\Lambda$ é fortemente simplesmente conexa.

(ii) Toda subcategoria plena convexa de $\Lambda$ tem a condição de separação.

(iii) Toda subcategoria plena convexa de $\Lambda^{o p}$ tem a condição de separação.

(iv) $H^{1}(C)=0$ para toda subcategoria plena convexa $C$ de $\Lambda$. 
Exemplo 1.39. Seja $\Lambda=\frac{k \Delta}{I}$ com

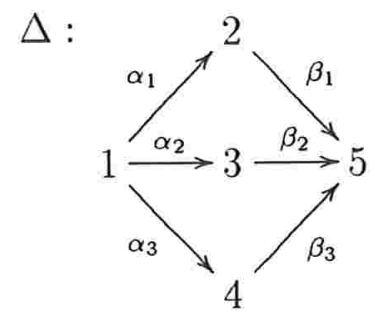

$\operatorname{com} I=<\sum_{i=1}^{3} \beta_{i} \alpha_{i}>$.

Temos que $\Lambda$ é uma álgebra fortemente simplesmente conexa.

A equivalência entre álgebras simplesmente conexas e fortemente simplesmente conexas em geral não ocorre. Vejamos o exemplo abaixo.

Exemplo 1.40. Seja $\Lambda=\frac{k \Delta}{I} \operatorname{com}$

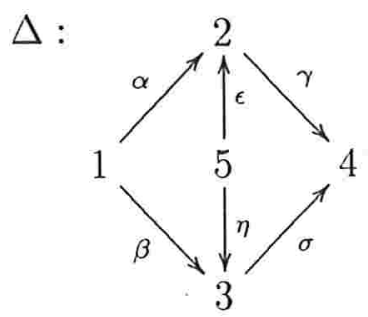

$\operatorname{com} I=<\gamma \alpha-\sigma \beta, \gamma \epsilon-\sigma \eta>$.

A álgebra $\Lambda$ é uma álgebra que é simplesmente conexa, mas não é fortemente simplesmente conexa (basta ver que o vértice 2 não é separado).

Uma classe de álgebras importante é a classe das álgebras inclinadas (ver [34]). Em 1.39 acima temos um exemplo de álgebra inclinada. Foi mostrado em [6] que se $\Lambda$ é uma álgebra inclinada fortemente simplesmente conexa então o grafo-órbita de cada uma das componentes conexas, pós-projetivas e pré-injetivas de $\Gamma_{\Lambda}$ é uma árvore. 
Em termos mais gerais foi mostrado em [31] que se $\Lambda$ é uma álgebra fortemente simplesmente conexa (não necessariamente inclinada), então o grafo-órbita de toda componente conexa dirigida de $\Gamma_{\Lambda}$ é uma árvore.

Em [8] foi obtida a seguinte caracterização das álgebras inclinadas mansas fortemente simplesmente conexas.

Teorema 1.41. Seja $\Lambda$ uma álgebra inclinada mansa. As seguintes condições são equivalentes:

(a) $\Lambda$ é fortemente simplesmente conexa.

(b) Os grafos-órbitas de cada uma das componentes pós-projetiva, pré-injetiva e conexa de $\Gamma_{\Lambda}$ é uma árvore.

(c) $H^{1}(\Lambda)=0$ e $\Lambda$ não contém subcategoria plena convexa que é hereditária de tipo $\tilde{\mathbf{A}}_{m}$.

(d) $\Lambda$ satisfaz a condição de separação e não contém subcategoria plena convexa que é hereditária de tipo $\tilde{\mathbf{A}}_{m}$. 


\section{Capítulo 2}

\section{Álgebras de tipo mesh}

Iremos estudar neste capítulo uma classe particular de álgebras, as álgebras de tipo mesh, que serão os nossos principais objetos de estudo ao longo deste trabalho. Estas álgebras generalizam as chamadas álgebras de Auslander, introduzidas em [9]. As álgebras de tipo mesh já haviam sido introduzidas na Teoria de Representações de Álgebras (ver, por exemplo [13], [40]). Até então as relações de tipo mesh consideradas eram as dadas por uma aljava de Auslander-Reiten. Logo, as álgebras de Auslander foram as álgebras de tipo mesh mais estudadas até agora. Consideraremos as álgebras de tipo mesh estudando-as em um contexto mais geral..

Na primeira seção iremos recordar a construção das álgebras de Auslander e alguns resultados importantes envolvendo tais álgebras. A seguir, na seção 2.2 , iremos definir as álgebras de tipo mesh e exibiremos alguns exemplos. Sempre pensando em comparar as álgebras de tipo mesh com as álgebra de Auslander, iremos nas seções seguintes discutir algumas propriedades das álgebras de tipo mesh. Na seção 2.4, em particular, iremos considerar um tipo especial de álgebras de tipo mesh definidas a partir das chamadas partições pós-projetivas.

O nosso estudo continua no capítulo seguinte quando analisaremos as álgebras de tipo mesh que são simplesmente conexas, fortemente simplesmente conexas e as relações com a cohomologia de Hochschild. 


\section{1 Álgebras de Auslander}

No estudo das álgebras de artin de tipo de representação finito, é muito útil considerarmos outra classe de álgebras de artin chamada álgebras de Auslander.

Definição 2.1. [13] Seja $\Lambda$ uma álgebra de tipo de representação finito, e $\left\{M_{1}, \ldots, M_{d}\right\}$ um conjunto completo de representantes de classes de isomorfia de $\Lambda$-módulos indecomponíveis. A álgebra $A=\operatorname{End}_{\Lambda}\left(\oplus_{i=1}^{d} M_{i}\right)$ é chamada a álgebra de Auslander $A$ de $\Lambda$.

Exemplo 2.2. Considere a álgebra $A=\frac{k \Delta}{I}$ onde

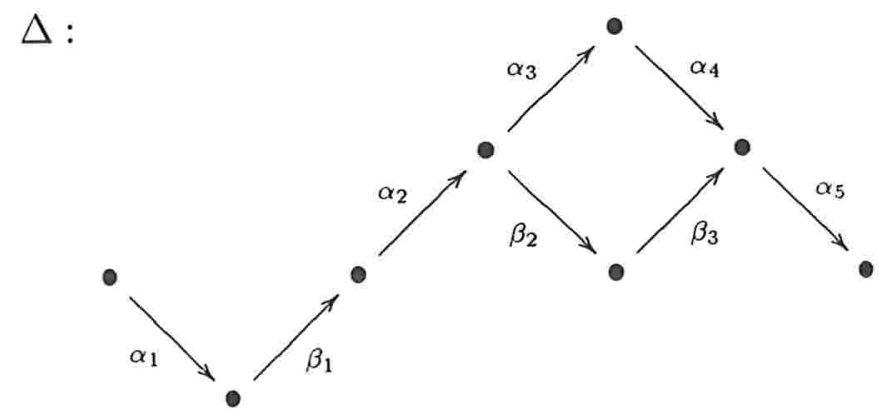

e $I$ é gerado por $\beta_{1} \alpha_{1}, \beta_{2} \alpha_{2}, \alpha_{4} \alpha_{3}-\beta_{3} \beta_{2}, \alpha_{5} \beta_{3}$.

A álgebra $A$ é a álgebra de Auslander de $\Lambda=\frac{k \Delta^{\prime}}{I^{\prime}}$ onde

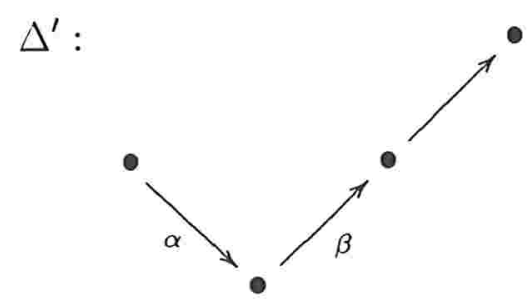

e $I^{\prime}$ é gerado por $\beta \alpha$.

Observe que se $A$ é a álgebra de Auslander de $\Lambda$, a aljava ordinária de $A$ coincide com a aljava de Auslander-Reiten de $\Lambda$. 
Por definição a álgebra de Auslander é sempre básica. Podemos observar também que uma álgebra básica semisimples é sempre uma álgebra de Auslander.

As álgebras de Auslander foram introduzidas inicialmente em [9] e são caracterizadas pelas propriedades homológicas do Teorema abaixo (para uma demostração ver por exemplo [27]).

Teorema 2.3. Uma álgebra A é uma álgebra de Auslander se e somente se satisfaz as seguintes condições:

(a) $g l \cdot \operatorname{dim} A \leq 2$.

(b) Se $0 \rightarrow A \rightarrow I_{0} \rightarrow I_{1} \rightarrow I_{2} \rightarrow 0$ é uma resolução injetiva minimal de $A$, então $I_{0}$ e $I_{1}$ são A-módulos projetivos.

Esta caracterização, por exemplo, é muito útil no desenvolvimento da teoria de recobrimento (Ver [16]).

Em [33], Happel estudou a cohomologia de Hochschild das álgebras de Auslander, fazendo também algumas comparações entre a cohomologia de Hochschild de $\Lambda$ e a cohomologia de Hochschild de sua álgebra de Auslander A. Vejamos alguns destes resultados:

Teorema 2.4. Seja A a álgebra de Auslander de $\Lambda$. Se $H^{1}(A)=0$, então $\Lambda$ é de representação direta (isto é, a aljava de Auslander-Reiten $\Gamma_{\Lambda}$ de $\Lambda$ é dirigida).

Teorema 2.5. Seja $\Lambda$ de tipo de representação finito e A sua álgebra de Auslander. Então $H^{1}(A)=0$ se e somente se $\Lambda$ é simplesmente conexa.

Teorema 2.6. Seja $\Lambda$ uma álgebra de tipo de representação finito e A sua álgebra de Auslander. Se $\Lambda$ é de representação direta, então $H^{2}(A)=0$.

Em [5], Assem e Brown caracterizaram as álgebras de Auslander fortemente simplesmente conexas e generalizaram o resultado de Happel em [33].

Teorema 2.7. Seja $\Lambda$ uma álgebra de tipo de representação finito, e seja A sua álgebra de Auslander. As seguintes afirmações são equivalentes: 
(a) $\Lambda$ é simplesmente conexa;

(b) A é simplesmente conexa;

(c) A é fortemente simplesmente conexa;

(d) $H^{1}(A)=0$;

(e) $H^{1}(C)=0$ para cada subcategoria plena convexa $C$ de $A$.

Observamos que podemos afirmar que a álgebra de Auslander $A$ de $\Lambda$ que apresentamos no exemplo 2.2 acima é simplesmente conexa e fortemente simplesmente conexa pois $\Lambda$ é uma álgebra simplesmente conexa.

\section{2 Álgebras de tipo mesh e exemplos}

Seja $\Delta$ uma aljava com translação. Uma relação de tipo mesh de um vértice $x$ em $\Delta$ é a combinação linear

$$
m_{x}=\sum_{\left\{\alpha \in \Delta_{1} / e(\alpha)=x\right\}} \alpha \sigma(\alpha)
$$

onde $\sigma$ é uma semitranslação fixada definida em $\Delta$.

Definição 2.8. Seja $\Delta$ uma aljava finita com translação. A álgebra $\Lambda=\frac{k \Delta}{\mu}$ onde $\mu$ é o ideal em $k \Delta$ gerado pelo conjunto das relações de tipo mesh é chamada a álgebra de tipo mesh associada a $\Delta$.

Obviamente, se $\Lambda$ é uma álgebra de tipo finito, então a álgebra de Auslander $A$ associada a $\Lambda$ é do tipo mesh. Como observado acima a aljava de Auslander-Reiten de $\Lambda$ é a aljava ordinária de $A$, isto é, $A=\frac{k \Gamma_{\Lambda}}{\mu}$.

Cabe observarmos aqui que neste caso, $\Gamma_{\Lambda}$ pode conter circuitos orientados mas mesmo assim a álgebra $A=\frac{k \Gamma_{\Lambda}}{\mu}$ é de dimensão finita sobre $k$. Isto é devido ao lema de Nakayama que estabelece a existência de um limite superior no comprimento das sequências de morfismos não isomorfismos entre indecomponíveis em uma álgebra 
de tipo finito. Com isto, as bases $B$ de caminhos de $\Gamma_{\Lambda}$ linearmente independentes módulo as relações mesh são finitas e portanto $A=\frac{k \Gamma_{\Lambda}}{\mu}$ é de dimensão finita sobre $k$.

No caso mais geral em que se começa com uma aljava finita com translação $\Delta$ nem sempre a álgebra de tipo mesh associada é de dimensão finita sobre $k$. As seguintes aljavas, por exemplo, nos fornecem álgebras de dimensão infinita.

Exemplo 2.9. $\Lambda=k \Delta$ com

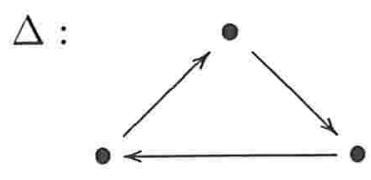

onde todos os vértices são projetivos e injetivos (e portanto, $\mu=0$ ).

Exemplo 2.10. $\Lambda=\frac{k \Delta}{\mu}$ com

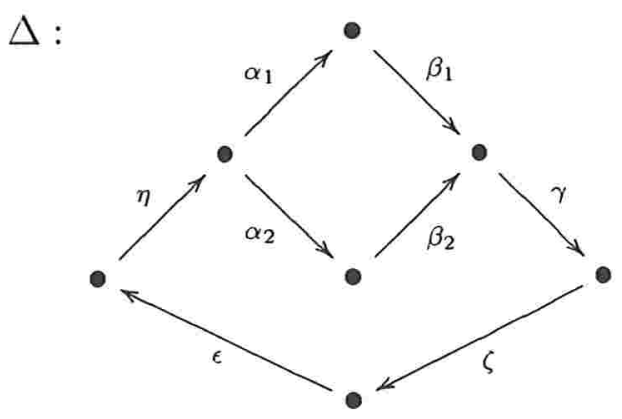

$\operatorname{com} \mu=<\beta_{1} \alpha_{1}-\beta_{2} \alpha_{2}>$.

Observamos que os caminhos $\left(\epsilon \zeta \gamma \beta_{2} \alpha_{2} \eta\right)^{n} \notin \mu, \forall n$.

No entanto, se $\Delta$ é uma aljava com translação finita e dirigida, então a álgebra de tipo mesh associada a $\Delta$ é de dimensão finita sobre $k$ pois neste caso, obviamente, $k \Delta$ tem uma base finita de caminhos. Por outro lado, a definição de aljava-órbita de uma aljava com translação depende essencialmente do fato de $\Delta$ ser dirigida e finita. Também a definição de álgebras simplesmente conexas faz sentido para álgebras triangulares, isto é, onde a aljava $\Delta$ associada seja dirigida. Por estes motivos iremos nos restringir neste trabalho ao estudo de álgebras de tipo mesh com aljava ordinária dirigida e finita. 
Temos como exemplos de álgebras de tipo mesh as álgebras hereditárias e as álgebras de Auslander que já citamos acima.

Alguns outros exemplos de álgebras de tipo mesh com a aljava ordinária dirigida:

Exemplo 2.11. $\Lambda=\frac{k \Delta}{\mu} \mathrm{com}$

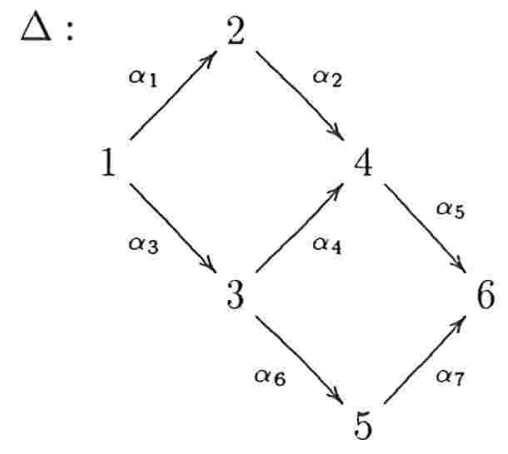

onde $\mu$ é o ideal gerado por $\alpha_{2} \alpha_{1}-\alpha_{4} \alpha_{3}$ e $\alpha_{5} \alpha_{4}-\alpha_{7} \alpha_{6}$.

$\Lambda$ é uma álgebra de tipo mesh que não é álgebra de Auslander.

Exemplo 2.12. $\Lambda=\frac{k \Delta}{\mu} \mathrm{com}$

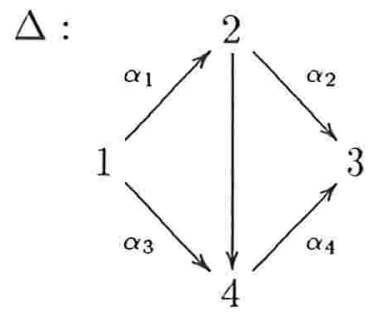

onde $\mu$ é o ideal gerado por $\alpha_{2} \alpha_{1}-\alpha_{4} \alpha_{3}$.

Este é um exemplo de uma álgebra de tipo mesh que possui componente pós-projetiva.

Continuaremos na próxima seção estudando algumas propriedades das álgebras de tipo mesh com a finalidade de descrevê-las e entendê-las melhor. 


\subsection{Algumas propriedades para as álgebras de tipo mesh}

Como as álgebras de tipo mesh formam uma classe de álgebras mais geral que a das álgebras de Auslander, é interessante estudá-las fazendo algumas comparações com as álgebras de Auslander. Por exemplo, não é verdadeiro que toda subcategoria plena $\mathcal{C}$ de uma álgebra de Auslander é uma álgebra de Auslander, mas $\mathcal{C}$ é uma álgebra de tipo mesh, vejamos o próxima resultado.

Proposição 2.13. Toda subcategoria plena $\mathcal{C}$ de uma álgebra de tipo mesh $\Lambda=\frac{k \Delta}{\mu}$ é também uma álgebra de tipo mesh $\mathcal{C}=\frac{k \Delta_{\mathcal{C}}}{I}$ onde $\Delta_{\mathcal{C}}$ é uma subaljava plena de $\Delta$.

\section{Prova:}

Ao considerarmos a álgebra $\Lambda$ como uma $k$-categoria $\mathcal{D}$ temos $\mathcal{D}=\left(D\right.$, mor $\left._{\mathcal{D}}, \circ\right)$ onde a classe dos objetos $D$ é o conjunto $\Delta_{0}$ e o conjunto mor ${ }_{\mathcal{D}}$ dos morfismos em $\mathcal{D}$ é para cada par $x, y$ em $D$ o espaço vetorial $k \Delta(x, y)$ de todas as combinações lineares de caminhos em $\Delta$ de $x$ para $y$ módulo o subspaço $\mu(x, y)=\mu \cap k \Delta(x, y)$ das relações de tipo mesh entre $x$ e $y$.

Observamos que o representa a operação de composta entre os caminhos de $\Delta$.

Vamos agora considerar $\mathcal{C}=\left(C\right.$, mor $_{\mathcal{C}}$, o $)$ uma subcategoria plena de $\mathcal{D}$. Neste caso $C \subseteq D, \operatorname{mor}_{\mathcal{C}}(x, y)=\operatorname{mor}_{\mathcal{D}}(x, y)$ para cada $x, y \in C$ e $\circ$ em $\mathcal{C}$ é a restrição de o em $\mathcal{D}$.

Primeiro observamos que $C=\Delta_{0}^{\prime}$ é um subconjunto de $\Delta_{0}$. A subcategoria $\mathcal{C}$ pode ser vista como uma álgebra de caminhos com relações $\frac{k \Delta^{\prime}}{I}$ onde $\Delta^{\prime}$ é uma subaljava plena de $\Delta$. (É plena pela definição de subaljava plena).

$\mathrm{O}$ conjunto mor $_{\mathcal{C}}$ dos morfismos em $\mathcal{C}$ é o espaço vetorial $k \Delta^{\prime}(x, y)$ de todas as combinações lineares de caminhos em $\Delta^{\prime}$ de $x$ para $y$ módulo o subspaço $I(x, y)=$ $I \cap k \Delta^{\prime}(x, y)$.

Com isto, o ideal $I$ é o ideal gerado pelas relações de tipo mesh da subaljava $\Delta^{\prime}$, o que prova o resultado. 
Observamos que se $\Lambda$ é uma álgebra de Auslander, podemos escrevê-la como uma extensão por um ponto $\Lambda=B[M]$, mas $B$ não deve ser uma álgebra de Auslander. O fato das álgebras de tipo mesh serem uma classe mais geral, obtemos para estas o resutado abaixo.

Proposição 2.14. Seja $\Lambda=\frac{k \Delta}{\mu}$ uma álgebra de tipo mesh onde $\Delta$ é uma aljava finita, dirigida e com translação . Então podemos escrever $\Lambda=B[M]$ onde $B$ é uma álgebra de tipo mesh e $M \in \bmod B$.

Prova: Temos que $\Lambda=\frac{k \Delta}{\mu}$ com $\Delta$ aljava finita com translação sem circuitos orientados. Então $\Lambda$ é de dimensão finita sobre $k$.

Como $\Delta$ é finita e dirigida existe pelo menos um poço em $\Delta$. Vamos tomar $z \mathrm{um}$ poço em $\Delta$ e $\bar{e}_{z}$ o idempotente correspondente em $\Lambda$. Como não existem caminhos não triviais saindo de $z$, temos $\bar{e}_{z} \Lambda \bar{e}_{z} \cong k$ e $\left(1-\bar{e}_{z}\right) \Lambda \bar{e}_{z}=0$.

Se $\left(\Delta^{\prime}, \rho\right)$ denota a aljava com relações que obtemos removendo o vértice $z$ e as relações terminando em $z$, então $\left(1-\overline{e_{z}}\right) \Lambda\left(1-\overline{e_{z}}\right) \cong k\left(\Delta^{\prime}, \rho\right)$. Portanto $\Lambda$ é obtida de $B=\frac{k \Delta^{\prime}}{\rho}$ adicionando o vértice $z$ e as flechas e relações de tipo mesh terminando em $z$. Temos então que

$$
\Lambda=\left(\begin{array}{ll}
B & 0 \\
M & k
\end{array}\right)
$$

onde $M=\left(1-\overline{e_{z}}\right) \Lambda \overline{e_{z}}=\operatorname{rad} P_{\Lambda}(z)$.

Assim, $\Lambda$ é a extensão por um ponto da álgebra $B$ por $M$ e escrevemos $\Lambda=B[M]$. Falta mostrar que se $\Lambda=B[M]$ é uma álgebra de tipo mesh, então $B$ é uma álgebra de tipo mesh.

$B=\frac{k \Delta^{\prime}}{\langle\rho\rangle}=\left(1-\overline{e_{z}}\right) \Lambda\left(1-\overline{e_{z}}\right)$.

1. $\Delta^{\prime}$ é uma aljava com translação e sem circuitos orientados.

De fato, claramente $\Delta^{\prime}$ não possui circuitos orientados, nem laços, pois $\Delta$ não os possui. Quanto a translação, se $\tau x$ está definida para $x$ em $\Delta_{0}$ então se $x$ está em $\Delta_{0}^{\prime}, \tau x$ está definida como a mesma translação de $\Delta$ (basta observarmos 
que ao retirarmos o vértice $z$ que é poço em $\Delta$ não retiramos de $\Delta_{0}$ nenhum transladado $\tau x$ de algum vértice $x$ de $\Delta_{0}^{\prime}$ ).

2. $\rho=0$ ou $\rho$ é gerado pelas relações de tipo mesh.

Temos obviamente que se $\Lambda=(\Delta, \mu)$ possui uma única relação de tipo mesh $m_{z}$ terminando no poço $z$ então $\rho=0$. Se $(\Delta, \mu)$ possui outras relações de tipo mesh $m_{x}$ para vértices $x \neq z$ estas relações de tipo mesh são preservadas em $\Delta^{\prime}$ pois $z$ é poço; logo não temos em $\Delta$ relações de tipo mesh nem começando e nem passando por $z$. Assim, as relações em $\rho$ são relações de tipo mesh.

Sobre a existência de componentes pós-projetivas existe a seguinte conjectura para as álgebras de Auslander:

Conjectura: As álgebras de Auslander possuem componente pós- projetiva.

Podemos então perguntar se as álgebras de tipo mesh possuem componente pósprojetiva. Os próximos exemplos mostram que isto não é verdade em geral:

Exemplo 2.15. Consideremos a álgebra de tipo mesh $\Lambda=\frac{k \Delta}{\mu}$ onde

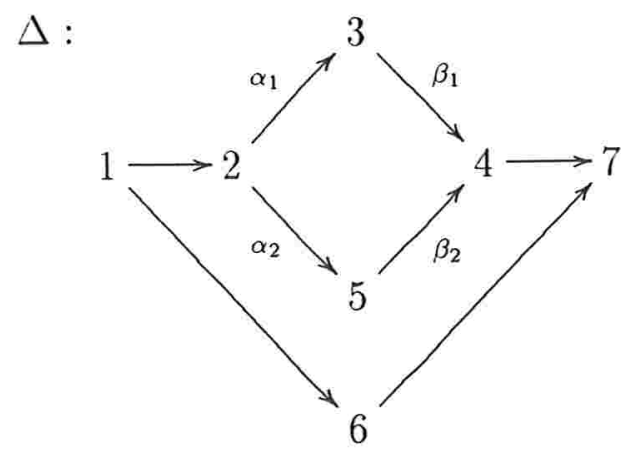

e $\mu=<\beta_{1} \alpha_{1}-\beta_{2} \alpha_{2}>$.

Temos que $\Lambda$ não satisfaz o Teorema 1.26, Capítulo 1 . De fato, basta vermos que $\Lambda=B[M]$ onde $M=\operatorname{rad} P_{7}$ não é dirigido como $B$-módulo pois existem somandos $P_{4}$ 
e $P_{6}$ de $M$ e $W=\operatorname{rad}_{4}$ indecomponível não projetivo tais que $P_{6} \preceq \tau W$ e $W \preceq P_{4}$. Logo $\Lambda$ não possui componente pós-projetiva.

Exemplo 2.16. Consideremos a álgebra de tipo mesh $\Lambda=\frac{k \Delta}{\mu}$ onde

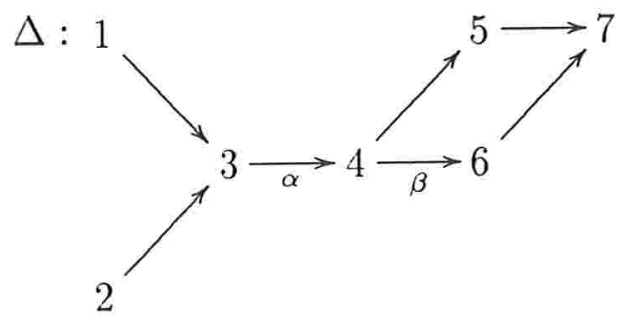

$\operatorname{com} \mu=<\beta \alpha>$.

Verificamos que $\Lambda$ não possui componente pós-projetiva usando novamente o critério de Dräxler - de la Peña ( ver Teorema 1.26 ). Basta vermos que $\Lambda=B[M]$ onde $M=\operatorname{rad} P_{7}$ não é dirigido como $B$-módulos pois existem somandos $P_{5}$ e $P_{6}$ de $\operatorname{rad} P_{7}$ e $W=\operatorname{rad} P_{6}$ não projetivo indecomponível tal que $P_{5} \preceq \tau W$ e $W \preceq P_{6}$.

Gostaríamos de salientar aqui que em termos homológicos as álgebras de tipo mesh podem distanciar-se bastante das álgebras de Auslander. Vimos no Teorema 2.3 que as álgebras de Auslander possuem dimensão global menor ou igual a 2. Mas as álgebras de tipo mesh não possuem tal propriedade. Vejamos os seguintes exemplos:

Exemplo 2.17. Vamos considerar a álgebra $\Lambda=\frac{k \Delta}{\mu} \operatorname{com}$

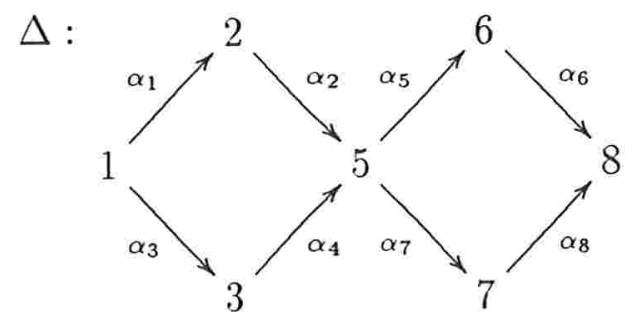

onde $\mu$ é o ideal gerado por $\alpha_{2} \alpha_{1}-\alpha_{4} \alpha_{3}, \alpha_{5} \alpha_{2}, \alpha_{7} \alpha_{4}, \alpha_{6} \alpha_{5}-\alpha_{8} \alpha_{7}$. Obtemos assim uma álgebra de tipo mesh que possui dimensão global 3 . 
Exemplo 2.18. Consideremos a álgebra $\Lambda=\frac{k \Delta}{\mu} \mathrm{com}$

$\Delta:$

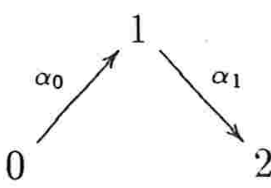

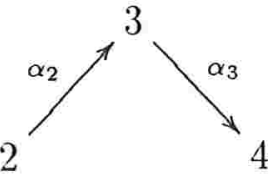

$\cdots$

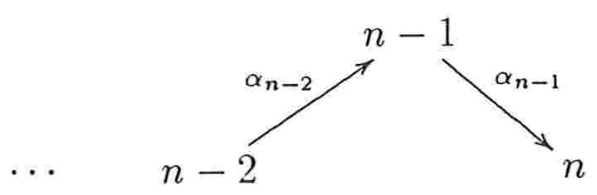

onde o ideal $\mu$ é gerado por $\alpha_{i+1} \alpha_{i}, 0 \leq i \leq n-2$.

Temos que $\Lambda$ é uma álgebra de tipo mesh de dimensão global $\mathrm{n}$.

Exemplo 2.19. Ao considerarmos a álgebra $\Lambda=\frac{k \Delta}{\mu} \operatorname{com} \Delta$ como no exemplo acima e ainda fazendo uma identificação entre os vértices 0 e $n ; \mu$ também como no exemplo acima, obtemos uma álgebra de tipo mesh $\Lambda$ com dimensão global infinita.

\subsection{Um caso particular de álgebras de tipo mesh}

Inicialmente vamos introduzir o conceito de partições pós-projetivas de ind $\Lambda$ onde $\Lambda$ é uma álgebra de artin (nesta seção, trabalhando com álgebras de artin estamos em um contexto mais geral do que o das álgebras de dimensão finita sobre um corpo algebricamente fechado) introduzido por Auslander e Smalø em [12].

Definição 2.20. Uma partição $\mathcal{P}_{i}$ de ind $\Lambda$ com $i$ em $\mathbb{N}_{\infty}=\mathbb{N} \cup\{\infty\}$ será chamada pós-projetiva quando as seguintes condições forem verdadeiras:

(a) ind $\Lambda$ é a união disjunta das subcategorias $\mathcal{P}_{i}$ onde $i \in \mathbb{N}_{\infty}$.

(b) para cada $j<\infty, \mathcal{P}_{j}$ é uma cobertura finita minimal da união das subcategorias $\mathcal{P}_{i} \operatorname{com} j \leq i \leq \infty$.

Observamos que uma cobertura para uma subcategoria $\mathcal{C}$ de ind $\Lambda$ é uma subcategoria $\mathcal{D}$ de $\mathcal{C}$ tal que para cada $X$ em $\mathcal{C}$ existe um epimorfismo de $Y \in$ addD para $X$. Ela é chamada minimal se nenhuma subcategoria própria de $\mathcal{D}$ tem a mesma propriedade.

Em [12] foi provado o seguinte resultado: 
Teorema 2.21. Seja $\Lambda$ uma álgebra de artin. Então existe uma única partição pósprojetiva de ind $\Lambda$.

Um $\Lambda$-módulo $M$ cujos somandos pertencem a união das subcategorias $\mathcal{P}_{i}$ com $i<\infty$ será chamado pós-projetivo. Se um módulo indecomponível $X$ está em $\mathcal{P}_{n}$, $0 \leq n \leq \infty$, então vamos denotar $n$ por $\pi(X)$. No caso em que $n<\infty$, iremos dizer que $X$ é um módulo pós-projetivo de nível $n$. Vamos denotar como $\mathcal{P}^{m}$ a subcategoria dos $\Lambda$-módulos pós-projetivos indecomponíveis até o nível $m$.

Os módulo em $\mathcal{P}_{0}$ são os projetivos indecomponíveis. A seguinte Proposição caracteriza os módulos pós-projetivos de nível 1.

Proposição 2.22. ([12]) Um módulo indecomponivel $X$ é um pós-projetivo de nível 1 se e somente se $X$ não é projetivo e existe uma aplicação irredutível de um projetivo para $X$.

Sendo $\Lambda$ uma álgebra de artin, vamos definir a álgebra $\Lambda_{s}=\operatorname{End} \oplus_{M \in P^{s}} M$ onde $0 \leq s<\infty$.

Observamos que quando $\Lambda$ é uma álgebra de tipo de representação finito, então a subcategoria $\mathcal{P}_{\infty}=\varnothing$. Então, existe $n$ tal que $i n d \Lambda=\cup_{i=0}^{n} \mathcal{P}_{i}$. Deste modo, a álgebra $\Lambda_{n}$ coincide com a álgebra de Auslander de $\Lambda$.

Em [45] Todorov e Zacharia observaram que se $\Lambda$ é uma álgebra de tipo de representação finito tal que sua álgebra de Auslander é de tipo de representação finito, então $\alpha(M) \leq 2$ para todo $\Lambda$-módulo indecomponível $M$ onde $\alpha(M)$ denota o número de somandos indecomponíveis de $E$, onde $E \rightarrow M$ é um poço.

Vamos agora estudar a classe das álgebra $\mathcal{H}_{n}, n \in \mathbb{N}$ introduzida por $\mathrm{F}$. Coelho em [21]. A classe $\mathcal{H}_{0}$ é a classe das álgebras hereditárias.

Definição 2.23. Dizemos que a álgebra $\Lambda$ está em $\mathcal{H}_{n}$ para algum $n$ se $\Lambda$ satisfaz as seguintes condições para $j=n$ mas não para $j<n$.

$\left(I_{j}\right)$ AddP $^{j}$ é fechado para submódulos. 
$\left(I I_{j}\right)$ Para toda aplicação irredutivel de $X$ em ind $\Lambda$ para $Y$ em $\mathcal{P}_{j+1}$ temos $\pi(X) \geq j$.

Algumas considerações:

- A condição $\left(I I_{0}\right)$ é trivialmente verdadeira pois todos os módulos indecomponíveis $X$ satisfazem $\pi(X) \geq 0$. Então $\Lambda$ está em $\mathcal{H}_{0}$ se e somente se $a d d \mathcal{P}_{0}$ é fechado para submódulos e isto é equivalente a uma álgebra ser hereditária.

- A condição $\left(I I_{1}\right)$ segue de $\left(I_{1}\right)$. De fato, suponha que $Y$ é um módulo em $\mathcal{P}_{2}$ e que existe uma aplicação irredutivel de $X$ em ind $\Lambda$ para $Y$. Se $\pi(X)<1$ então $X$ é um módulo projetivo e $Y$ tem que estar em $\mathcal{P}^{1}$ pela Proposição acima e isto é uma contradição.

Teorema 2.24. ([21]): Seja $\Lambda$ uma álgebra em $\mathcal{H}_{n}$ para algum n. Então para todo $m \geq n$ as condições $\left(I_{m}\right)$ e $\left(I I_{m}\right)$ também valem.

Corolário 2.25. ([21]): Seja $\Lambda$ uma álgebra em $\mathcal{H}_{n}$ para algum $n$ e $X \rightarrow Y$ uma aplicação irredutível. Então

1. Se $\pi(Y) \leq n$ então $\pi(X) \leq n$,

2. Se $\pi(Y)=n+1$ então $\pi(X) \leq n+1$,

3. Se $\pi(Y)>n+1$ então $\pi(Y)-1 \leq \pi(X) \leq \pi(Y)$.

Seja agora $\Lambda \in \mathcal{H}_{n}$ para algum $n \geq 0$ e $s \geq n$. Segue dos resultados acima que os módulos de $\bigcup_{i \leq s} \mathcal{P}_{i}$ determinam uma subaljava conexa de $\Gamma_{\Lambda}$ e portanto $\Lambda_{s}$ é uma álgebra de tipo mesh.

Em [1] Algeleri-Hügel e Coelho mostraram que se $\Lambda$ é álgebra conexa em $\mathcal{H}_{1}$ então ou $\Lambda$ é álgebra com radical quadrado zero com aljava $\tilde{\mathbf{A}}_{n}$ linearmente ordenada ou $\Gamma_{\Lambda}$ tem uma componente pós-projetiva contendo todos os módulos projetivos indecomponíveis. No segundo caso, teremos que $\Lambda_{s}$ é álgebra triangular para todo $s \geq 1$. Para álgebras $\Lambda \in \mathcal{H}_{n}, \operatorname{com} n \geq 2$, a situação é um pouco mais complexa. 
Exemplo 2.26. Seja $\Lambda=\frac{k \Delta}{\mu}$ a álgebra dada pela aljava

$$
\Delta: \bullet \underset{\alpha_{2}}{\stackrel{\alpha_{1}}{\longrightarrow}} \bullet \stackrel{\beta}{\longrightarrow} \bullet \curlyvee
$$

$\operatorname{com} \mu=<\gamma \beta, \beta \alpha_{1}, \beta \alpha_{2}>$.

A componente conexa de $\Gamma_{\Lambda}$ contendo os módulos projetivos é como segue:

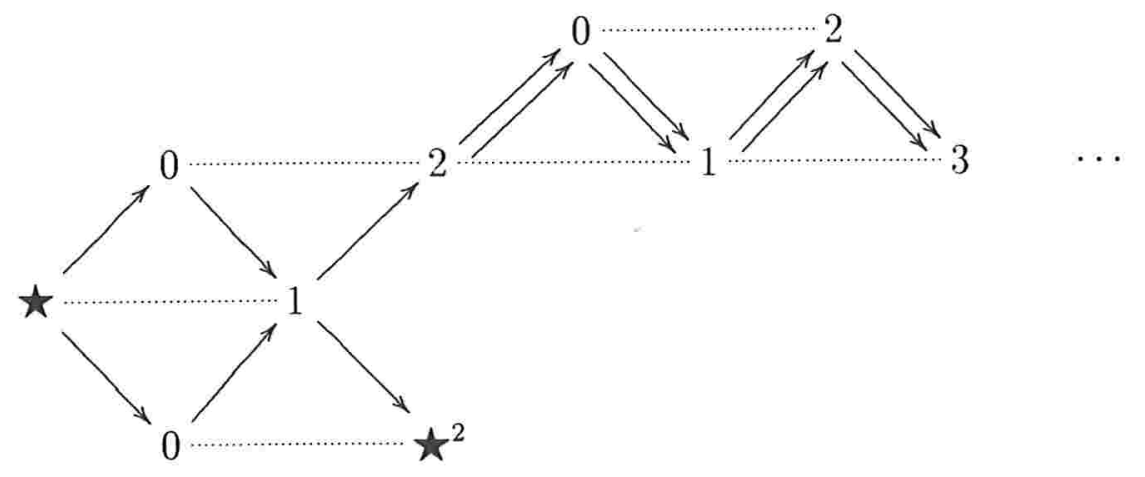

onde devem ser identificados os módulos marcados com $\star$. O número dado a cada vértice identifica o nível pós-projetivo do módulo correspondente. Não é difícil ver que $\Lambda \in \mathcal{H}_{2}$.

Observe então que $\Lambda_{2}$ é dada pela aljava com translação

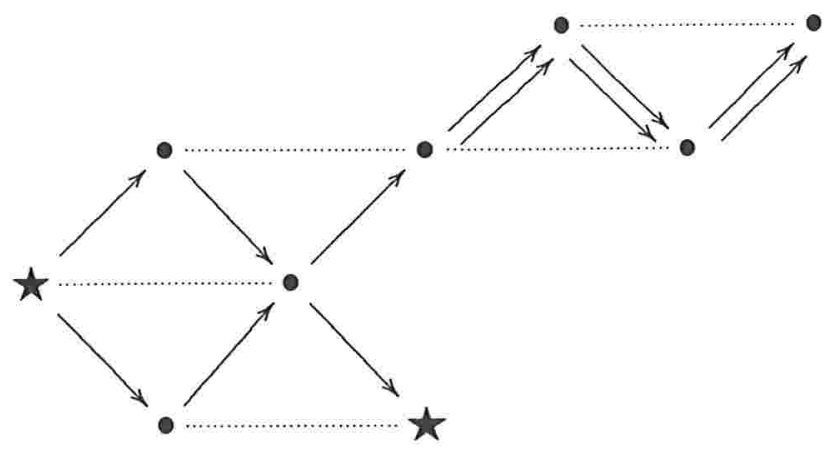

onde os vértices marcados por $\star$ devem ser identificados. É claro que $\Lambda_{2}$ não é uma álgebra triangular.

Como vimos na seção 2.3, as álgebras de tipo mesh não possuem, em geral, uma limitação para a dimensão global. Veremos que para uma classe especial de 
álgebras de tipo mesh podemos obter uma limitação para a sua dimensão global. Consideremos a álgebra $\Lambda_{s}=\operatorname{End}_{\Lambda}(P)$ onde $P=\oplus_{M \in \mathcal{P}} \boldsymbol{M}$. S. Gastamiza mostrou em [30] que se $\Lambda$ é uma álgebra de artin hereditária então gldim $\Lambda_{s}=2$ para cada $s$ tal que $\mathcal{P}_{s}$ é não vazio, $0<s<\infty$.

Iremos tratar aqui de uma generalização deste resultado, para isto, inicialmente precisaremos do seguinte lema.

Lema 2.27. Se $\Lambda_{s}=\operatorname{End}_{\Lambda}(P)$ como acima.Então temos que:

1. $P$ é um $\Lambda_{s}$-módulo projetivo à direita.

2. Os funtores $F: N \rightarrow \operatorname{Hom}_{\Lambda}(P, N)$ e $G: M \rightarrow M \otimes P$ estabelecem uma equivalência entre a categorias addP $\mathcal{P}^{s}$ e a categoria dos $\Lambda_{s}$-módulos projetivos à direita que denotaremos por $\operatorname{pr} \Lambda_{s}$.

\section{Prova:}

1) Como $P=\oplus_{M \in \mathcal{P}}: M$, podemos escrever $P=\Lambda \oplus L$ para algum $L$.

$\operatorname{Logo}, \Lambda_{s}=\operatorname{End}_{\Lambda}(P) \cong \operatorname{Hom}_{\Lambda}(P, P) \cong \operatorname{Hom}_{\Lambda}(\Lambda, P) \oplus \operatorname{Hom}_{\Lambda}(L, P)$.

Portanto $\operatorname{Hom}_{\Lambda}(\Lambda, P) \cong P$ sendo um somando direto de $\Lambda_{s}$ é um $\Lambda_{s}$-módulo projetivo à direita.

2)Primeiro vemos que $F(N)$ é sempre um $\Lambda_{s}$-módulo projetivo à direita.

De fato, se tomamos $N \in a d d \mathcal{P}^{s}$, temos que $M$ é soma direta de somandos de $\mathcal{P}^{s}=\sum_{i} U_{i}^{n_{i}}$. Tomamos o $n_{i}$ máximo e o denotamos por $m$.

Logo, $m P=m N \oplus U$ para algum $U$. Então $m \Lambda_{s}=m E n d_{\Lambda}(P) \cong \operatorname{Hom}_{\Lambda}(m P, P) \cong$ $\operatorname{Hom}_{\Lambda}(m N, P) \oplus \operatorname{Hom}_{\Lambda}(U, P) \cong m F(N) \oplus \operatorname{Hom}_{\Lambda}(U, P)$.

Portanto $F(N)$ é um $\Lambda_{s}$-módulo projetivo pois é somando de um $\Lambda_{s}$-módulo livre.

Para concluir que as transformações naturais $\psi: G F \rightarrow a d d \mathcal{P}^{s}$ e $\varphi: F G \rightarrow \operatorname{pr} \Lambda_{s}$ são isomorfismos basta usar o Teorema de Morita. 
Teorema 2.28. Seja uma álgebra de artin $\Lambda \in \mathcal{H}_{n}, n \geq 0$. Suponhamos $\Lambda_{s}=$ $\operatorname{End}_{\Lambda}(P) \operatorname{com} \infty>s \geq n$ (quando $n=0$, a desigualdade é estrita). Então gldim $\Lambda_{s}=$ 2 .

Prova: Inicialmente vamos mostrar que $\operatorname{gldim} \Lambda_{s} \leq 2$. Seja $X \in i n d \Lambda_{s}$ não projetivo e consideremos uma apresentação projetiva para $X$ :

$$
P_{1} \stackrel{f}{\longrightarrow} P_{0} \longrightarrow X \longrightarrow 0 \text {. }
$$

Pela equivalência dada por $F=\operatorname{Hom}_{\Lambda}\left(P\right.$, ) (ver o Lema 2.27) temos que $P_{1}=$ $F\left(N_{1}\right), P_{0}=F\left(N_{0}\right)$ com $V_{1}$ e $N_{0}$ em $a d d \mathcal{P}^{s}$ e que existe $g: N_{1} \rightarrow N_{0}$ tal que $f=F(g)$.

Consideremos a sequência

$$
0 \longrightarrow \operatorname{kerg} \longrightarrow N_{1} \stackrel{g}{\longrightarrow} N_{0}
$$

Como $\Lambda \in \mathcal{H}_{n}$ temos que $a d d \mathcal{P}^{s}$ é fechado para submódulos com $s \geq n$. Então, como $N_{1} \in \operatorname{add} \mathcal{P}^{s}$, temos que $k e r g \in \operatorname{add} \mathcal{P}^{s}$.

Podemos agora aplicar o funtor $F$ que é exato à esquerda na sequência 2.1 e obtemos:

$$
0 \longrightarrow F(\text { ker } g) \longrightarrow F\left(N_{1}\right) \longrightarrow F\left(N_{0}\right) \longrightarrow X \longrightarrow 0
$$

onde $F($ ker $g)$ é um $\Lambda_{s}$-módulo projetivo.

Logo, $g l \operatorname{dim} \Lambda_{s} \leq 2$. Neste caso, temos que $g l \operatorname{dim} \Lambda_{s}=2$. De fato, como nossa álgebra é $\Lambda_{s}$ com $s \geq 1$, temos que existe pelo menos um vértice que não é projetivo e isto implica que ocorrerá pelo menos uma relação de tipo mesh na aljava ordinária de $\Lambda_{s}$. Logo, $\Lambda_{s}$ não é uma álgebra hereditária, então gldim $\Lambda_{s} \geq 2$. Concluímos pelo que provamos anteriormente que $\Lambda_{s}$ deve ter dimensão global igual a dois.

Observamos que o caso $\Lambda \in \mathcal{H}_{0}$, a álgebra $\Lambda_{0}$ coincide com a álgebra $\Lambda$, portanto gldim $\Lambda_{0} \leq 2$.

Temos assim que as álgebras $\Lambda_{s} \operatorname{com} \Lambda \in \mathcal{H}_{n}$ e $s \geq n$ são exemplos de álgebras de tipo mesh que aproximam-se das álgebras de Auslander por terem uma propridade homológica em comum. 
Com o resultado a seguir obtemos uma condição necessária para que a álgebra de tipo mesh $\Lambda_{s}$ seja de tipo de representação finito.

Teorema 2.29. Seja $\Lambda$ uma álgebra de artin em $\mathcal{H}_{n} \operatorname{com} n \geq 0$. Se $\Lambda_{s}$ é de tipo de representação finito para algum $s \geq n$ (no caso $n=0$, considere a desigualdade estrita) então $\alpha(M) \leq 2$ para todo $\Lambda$-módulo indecomponível $M$.

Prova: Observe inicialmente que como $\Lambda_{s}$ é de tipo de representação finito, então $\Lambda$ é de tipo de representação finito. Portanto temos que $\mathcal{P}_{\infty}$ é vazio.

Vamos tomar um $\Lambda$-módulo indecomponível $M$ e provar por indução em $m=$ $\pi(M)$ que $\alpha(M) \leq 2$.

Vejamos o caso em que $m=0$, ou seja, $M$ é um $\Lambda$-módulo projetivo. Suponhamos por contradição que $\alpha(M)=t \geq 3$. Então ao considerarmos o morfismo minimal à direita $\mathrm{rad} M \rightarrow M$ temos que $\mathrm{rad} M=E_{1} \oplus E_{2} \oplus \cdots \oplus E_{t}$. Como $M$ é projetivo, os morfismos $E_{i} \rightarrow M \operatorname{com} i=1,2, \ldots, t$ são monomorfismos. Desta maneira, cada $E_{i}$ é não injetivo, logo existem $\tau^{-1} E_{i} i=1,2, \ldots, t$. Pelo Corolário 2.25 temos que $\pi\left(E_{i}\right) \leq n$. Pela Proposição 2.22 temos que $\pi\left(\tau^{-1} E_{i}\right)=1$ com $i=1,2, \ldots, t$ pois $M$ é projetivo.

Assim, obtemos a seguinte subaljava de $\Lambda_{s}$ que claramente é de tipo de representação infinito:

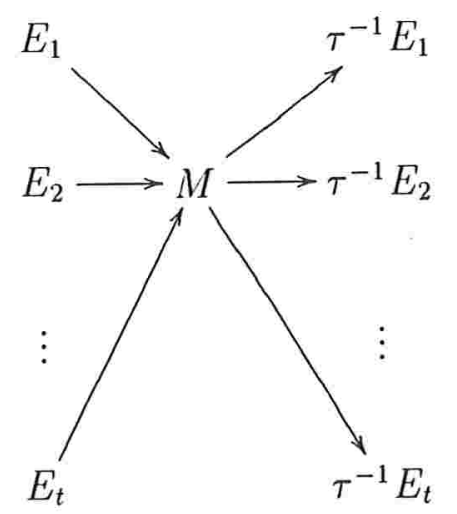

Então $\Lambda_{s}$ deve ser de tipo de representação infinito para todo $s \geq n$ o que é uma contradição.

Vamos considerar o caso em que $\pi(M)=m$ com $1 \leq m \leq n$. Suponhamos 
por contradição que $\alpha(M)=t \geq 3$. Consideremos o morfismo minimal à direita $E \rightarrow M \rightarrow 0$, então neste caso $E=E_{1} \oplus E_{2} \oplus \cdots \oplus E_{t}$ e como $M$ é não projetivo temos que existe $\tau M$. Pelo Corolário 2.25 temos que $\pi\left(E_{i}\right) \leq n$ para $i=1, \ldots, t$. Como consequência disto temos a seguinte subaljava de $\Lambda_{s}$ que é conhecida ser de tipo de representação infinito :

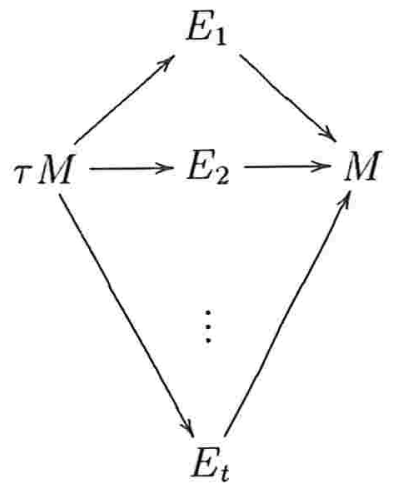

Então $\Lambda_{s}$ deve ser de tipo de representação infinito para todo $s \geq n$ o que é uma contradição.

Assumimos agora $\pi(M) \geq n+1$.

Suponhamos por hipótese de indução que se $\Lambda_{s}$ é de tipo de representação finito para algum $s \geq n$ então $\alpha(M) \leq 2$ para todo $\Lambda$-módulo indecomponível com $\pi(M) \leq$ $m-1$.

Queremos mostrar que a afirmação vale para todo $\Lambda$-módulo $M \operatorname{com} \pi(M)=m$. Para este propósito suponhamos $\pi(M)=m$. Consideremos o morfismo minimal à direita $E \rightarrow M$. Suponhamos por contradição que $\alpha(M)=t \geq 3$, então $E=$ $E_{1} \oplus E_{2} \oplus \cdots \oplus E_{t}$.

Pelo Corolário 2.25 , temos que $m-1 \leq \pi\left(E_{i}\right) \leq m, i=1,2, \ldots, t$. Como $m \geq 2$ temos que existem $\tau M$ e $\tau E_{i}$ para $i=1, \ldots, t$ e isto é uma contradição a nossa hipótese pois pelo Corolário 2.25 temos que $\tau M$ deve estar em $\mathcal{P}_{m-2} \cup \mathcal{P}_{m-1}$. Logo, $\alpha(M) \leq 2$ para todo $\Lambda$-módulo indecomponível com $\pi(M)=m$. 


\section{Capítulo 3}

\section{Álgebras de tipo mesh fortemente simplesmente conexas em característica zero}

Sejam $k$ um corpo algebricamente fechado com característica 0 e $\Delta$ uma aljava finita, conexa, dirigida e com translação. Vamos considerar a álgebra de tipo mesh $\Lambda=\frac{k \Delta}{\mu}$ como definimos no Capítulo 2. Observamos que $\Lambda$ é uma $k$-álgebra de dimensão finita.

Neste Capítulo iremos caracterizar as álgebras de tipo mesh que são fortemente simplesmente conexas através do seguinte teorema:

Teorema 3.1. Seja $\Delta$ uma aljava finita, dirigida, com translação e sem flechas múltiplas $e \Lambda=\frac{k \Delta}{\mu}$ a álgebra de tipo mesh associada a $\Delta$. Então as seguintes afirmações são equivalentes:

(a) $\Lambda$ é fortemente simplesmente conexa.

(b) $H^{1}(\Lambda)=0$.

(c) $H^{1}(C)=0$ para toda subcategoria plena convexa $C$ de $\Lambda$. 
(d) Todo ciclo irredutivel é um contorno irredutivel, e todo contorno irredutível é do tipo

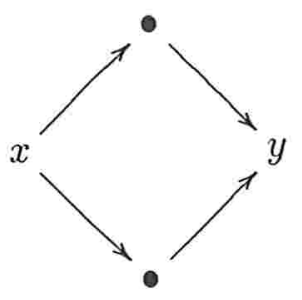

com $x=\tau y$.

(e) $\mathcal{O}(\Delta)$ é árvore.

(f) $\overline{\mathcal{O}}\left(\Delta^{\prime}\right)$ é árvore para toda subaljava $\Delta^{\prime} \subseteq \Delta$ de tipo $\tilde{\mathbf{A}}_{n}$.

(g) Toda subcategoria plena convexa $\mathcal{C}$ de $\Lambda$ satisfaz a condição de separação.

A equivalência (c) $\Rightarrow(b)$ é óbvia.

As equivalências (c) $\Leftrightarrow(\mathrm{a}) \Leftrightarrow(\mathrm{g})$ valem pelo Teorema 1.38 de Skowroński onde as álgebras fortemente simplesmente conexas foram caracterizadas pela propriedade de separação e pelo anulamento dos primeiros grupos de Cohomologia de Hochshild.

A implicação (d) $\Rightarrow$ (a) segue direto do Teorema 3.5 de Assem e Liu em ( [7]): Na seção 3.1 tratamos dos resultados que relacionam o primeiro grupo de cohomologia de Hochschild da álgebra de tipo mesh $\Lambda=\frac{k \Delta}{\mu}$ com a aljava-órbita $\mathcal{O}(\Delta)$. Com estes resultados concluímos a equivalência $(\mathrm{b}) \Leftrightarrow(\mathrm{e})$.

Na seção 3.2 apresentaremos os conceitos de ciclos e contornos irredutíveis e a caracterização que Assem e Liu deram para as álgebras fortemente simplesmente conexas utilizando estes conceitos. Eles serão bastante úteis pela maneira de como se apresentam no item (d) do Teorema 3.1 sendo uma propriedade interessante e fácil de ser verificada em uma aljava finita, conexa, dirigida e com translação. Assim, nesta seção obteremos os resultados necessários para demonstrarmos a implicação (f) $\Rightarrow$ (d).

$\mathrm{Na}$ seção 3.3 concluiremos a demonstração do Teorema 3.1. E finalmente na seção 3.4 consideraremos algumas consequências e importância deste resultado. 


\subsection{Cohomologia de Hochschild para álgebras de tipo mesh}

Os resultados que iremos tratar nesta seção estendem para as classes de álgebras de tipo mesh alguns resultados que D. Happel provou em [33] para as álgebras de Auslander.

Até pouco tempo atrás, conjecturava-se que se uma álgebra $\Lambda=\frac{k \Delta}{I}$ satisfazia $H^{1}(\Lambda)=0$, então $\Delta_{\Lambda}$ não tinha circuitos orientados. Recentemente Buchweitz e Liu em [19] exibiram um exemplo mostrando a falsidade desta conjectura. O resultado abaixo nos mostra que para álgebras de tipo mesh a conjectura é verdadeira.

Teorema 3.2. Sejam $\Delta$ uma aljava finita, conexa, com translação $e \Lambda=\frac{k \Delta}{\mu} a$ álgebra de tipo mesh associada a $\Delta$. Se $H^{1}(\Lambda)=0$, então $\Delta$ não possui circuitos orientados e nem subaljava do tipo $\tilde{\mathrm{A}}_{1, p}, p \geq 2$.

\section{Prova:}

Denotamos $l(\gamma)$ o comprimento de um caminho $\gamma$ em $\Delta$ e

Definimos

$$
\delta: k \Delta \longrightarrow k \Delta
$$

e estendemos por linearidade.

Fato 1: $\delta$ é uma derivação normalizada (isto é, está em $\operatorname{Der}^{n}(k \Delta)$ ).

Inicialmente observamos que $\delta$ definida como acima pertence ao $k$-espaço vetorial das derivações de $k \Delta$ em $k \Delta$ pois $\delta \in \operatorname{Hom}_{k}(k \Delta, k \Delta)$ e para $b_{1}, b_{2} \in k \Delta$ temos:

Se $b_{1} b_{2} \neq 0, \delta\left(b_{1} b_{2}\right)=l\left(b_{1} b_{2}\right) b_{1} b_{2}=\left(l\left(b_{1}\right)+l\left(b_{2}\right)\right) b_{1} b_{2}=\delta\left(b_{1}\right) b_{2}+b_{1} \delta\left(b_{2}\right)$.

Se $b_{1} b_{2}=0, \delta\left(b_{1} b_{2}\right)=l\left(b_{1} b_{2}\right) b_{1} b_{2}=0=\left(l\left(b_{1}\right)+l\left(b_{2}\right)\right) b_{1} b_{2}=\delta\left(b_{1}\right) b_{2}+b_{1} \delta\left(b_{2}\right)$.

Sendo $\left\{e_{1}, \ldots, e_{n}\right\}$ um sistema completo de idempotentes primitivos e ortogonais de $\Lambda$, vale que $\delta\left(e_{i}\right)=l\left(e_{i}\right) e_{i}=0$ para todo $i=1, \ldots, n$. Logo, $\delta \in \operatorname{Der}^{n}(k \Delta)$.

Fato 2: $\delta(\mu) \subseteq \mu$.

Para cada vértice $x$ em $\Delta$ seja $m_{x}$ a relação de tipo mesh na aljava $\Delta$ definida por $m_{x}=\sum_{\left\{\alpha \in \Delta_{1} / e(\alpha)=x\right\}} \alpha \sigma(\alpha)$ onde $\sigma(\alpha)$ é a semitranslação de $\alpha$.

$\delta\left(m_{x}\right)=\sum_{\alpha} \delta(\alpha \sigma(\alpha))=2 \sum_{\alpha} \alpha \sigma(\alpha)$. 
Portanto, $\delta$ induz uma derivação normalizada $\bar{\delta} \in \operatorname{Der}^{n}(\Lambda), \bar{\delta}: \frac{k \Delta}{\mu} \longrightarrow \frac{k \Delta}{\mu}$.

Por hipótese temos que $0=H^{1}(\Lambda)=\frac{D r^{n}(\Lambda)}{D e r^{n, 0}(\Lambda)}$. Então existe $\lambda \in \Lambda$ tal que $\bar{\delta}=$ $\delta_{\lambda}$. Como $\bar{\delta} \in \operatorname{Der}^{n}(\Lambda)$, concluímos que $\lambda=\sum_{i=1}^{n} \mu_{i} e_{i}+\lambda^{\prime}$ onde $\mu_{i} \in k$ e $\lambda^{\prime} \in$ $\oplus_{i} e_{i}(\operatorname{rad} \Lambda) e_{i}$ (basta usar que $\left.\delta_{\lambda}\left(e_{i}\right)=0 \forall i\right)$.

Consideramos $\alpha$ uma flecha de $x_{i}$ para $x_{j}$ em $\Delta$ e denotamos por $\bar{\alpha}$ a sua classe residual em $\Lambda$. Então $\bar{\alpha}=\bar{\delta}(\bar{\alpha})=\bar{\delta}_{\lambda}(\bar{\alpha})=\lambda \bar{\alpha}-\bar{\alpha} \lambda=\mu_{i} \bar{\alpha}-\bar{\alpha} \mu_{j}+\tilde{\lambda}$ com $\tilde{\lambda} \in \operatorname{rad}^{2} \Lambda$. Daí $\bar{\alpha}=\left(\mu_{i}-\mu_{j}\right) \bar{\alpha}+\tilde{\lambda}$ de onde segue que $\mu_{i}-\mu_{j}=1$.

Suponhamos que $x_{1} \longrightarrow x_{2} \longrightarrow \cdots \longrightarrow x_{r} \longrightarrow x_{r+1}=x_{1}$ seja um circuito orientado em $\Delta$. Neste caso teremos que

$$
\mu_{i}-\mu_{i+1}=1 \quad \text { para } \quad 1 \leq i<r \quad \text { e } \quad \mu_{r}-\mu_{1}=1 .
$$

Agora, vamos considerar a seguinte subaljava do tipo $\tilde{\mathbf{A}}_{1, p}$ em $\Delta$ :

$$
x_{1} \longrightarrow x_{2} \longrightarrow \cdots \longrightarrow x_{r} \longleftarrow x_{r+1}=x_{1}
$$

Disto, temos que

$$
\mu_{i}-\mu_{i+1}=1 \quad \text { para } \quad 1 \leq i<r \quad \text { e } \quad \mu_{1}-\mu_{r}=1 .
$$

Chegamos a uma contradição pois os sistemas de equações lineares 3.1 e 3.2 não têm solução para $k$ com característica 0 .

Mencionamos também o seguinte Corolário que é interessante por si só.

Corolário 3.3. Sejam $\Delta$ uma aljava finita, conexa, dirigida, com translação e $\Lambda=$ $\frac{k \Delta}{\mu}$ a álgebra de tipo mesh associada $a \Delta$. Se $\Lambda$ é fortemente simplesmente conexa então $\Delta$ não possui flechas múltiplas.

Prova: Suponhamos que existam dois vértices $x_{1}$ e $x_{2}$ em $\Delta$ com flechas múltiplas de $x_{1}$ para $x_{2}$. Ao considerarmos a subaljava $\Delta^{\prime}$ formada por estes dois vértices $x_{1}$ e $x_{2}$ e todas as flechas de $x_{1}$ para $x_{2}$, afirmamos que esta é uma subaljava plena convexa de $\Lambda$. De fato, se isto não ocorre, deve existir pelo menos um caminho de $x_{1}$ para $x_{2}$ de comprimento maior ou igual a dois. Assim obtemos uma subaljava 
do tipo $\tilde{\mathbf{A}}_{1, p}, p \geq 2$ em $\Delta$. Pelo Teorema 3.2 , temos $H^{1}(\Lambda) \neq 0$ e isto contradiz o fato de $\Lambda$ ser fortemente simplesmente conexa (ver Teorema 1.38 de Skowroński).

Mas, por outro lado, ao considerarmos a subcategoria plena convexa $C$ associada $\Delta^{\prime}$ vemos que esta não é simplesmente conexa e isto também contradiz o fato de $\Lambda$ ser simplesmente conexa. Logo, $\Delta$ não possui flechas múltiplas.

Teorema 3.4. Sejam $\Delta$ uma aljava finita, conexa, dirigida, com translação e $\tilde{\mathbf{A}_{1, p}}$ - livre e $\Lambda=\frac{k \Delta}{\mu}$ a álgebra de tipo mesh associada a $\Delta$. Então $H^{1}(\Lambda) \cong H^{1}(k \mathcal{O}(\Delta))$ onde $\mathcal{O}(\Delta)$ é a aljava-órbita de $\Delta$.

\section{Prova:}

Vamos denotar o conjunto $\Delta_{0}=\left\{e_{1}, \ldots, e_{n}\right\}$. Como $\Delta$ é $\tilde{A_{1, p}}$ - livre segue que $\operatorname{dim}\left(e_{j} \Lambda e_{i}\right) \leq 1$ para todos $e_{i}, e_{j} \in \Delta_{0}$ tais que exista uma flecha de $e_{i}$ para $e_{j}$ em $\Delta$. Tomamos $\delta \in \operatorname{Der}^{n}(\Lambda)$.

Afirmação 1: Para cada $\alpha \in \Delta_{1}$, existe $\lambda_{\alpha} \in k$ tal que $\delta(\alpha)=\lambda_{\alpha} \alpha$.

Observe que se $\alpha: e_{i} \rightarrow e_{j}$, então teremos

$\delta(\alpha)=\delta\left(e_{j} \alpha\right)=\delta\left(e_{j}\right) \alpha+e_{j} \delta(\alpha)$ e

$\delta(\alpha)=\delta\left(\alpha e_{i}\right)=\delta(\alpha) e_{i}+\alpha \delta\left(e_{i}\right)$

Comparando estas expressões, segue que $e_{j} \delta(\alpha)=\delta(\alpha) e_{i}=\delta(\alpha)$.

Logo $\delta(\alpha) \in e_{j} \Lambda e_{i}$ que é gerado por $\alpha$. Como $\operatorname{dim}\left(e_{j} \Lambda e_{i}\right)=1$ temos que $\delta(\alpha)$ é gerado por $\alpha$ pelo observado acima, de onde segue o resultado.

Pela construção da aljava $\mathcal{O}(\Delta)$, podemos identificar as flechas de $\mathcal{O}(\Delta)$ com as flechas de $\Delta$ que chegam nos vértices projetivos. Iremos utilizar tal identificação livremente abaixo.

$\begin{array}{cccc}\text { Vamos definir agora } & \psi: \operatorname{Der}^{n}(\Lambda) & \longrightarrow & \operatorname{Der}^{n}(k \mathcal{O}(\Delta)) \\ \delta & \longmapsto & \bar{\delta}\end{array}$ definindo $\bar{\delta}(\alpha)=\delta(\alpha)$ para uma flecha $\alpha$ em $(\mathcal{O}(\Delta))_{1}$.

Afirmação $2: \psi$ é sobrejetora.

Dado $\delta \in \operatorname{Der}^{n}(k \mathcal{O}(\Delta))$, definiremos a seguir $\tilde{\delta} \in \operatorname{Der}^{n}(\Lambda)$ tal que $\psi(\tilde{\delta})=\delta$.

Para $e_{i} \in \Delta_{0}$, colocamos $\tilde{\delta}\left(e_{i}\right)=0$. 
Seja $x$ um vértice em $\Delta_{0}$, vamos definir $\tilde{\delta}(\beta)$ para toda flecha $\beta$ tal que $e(\beta)=x$ usando indução em $n=n(x)=$ o número de predecessores próprios de $x$.

Se $x$ for projetivo e $\alpha: y \rightarrow x$ então podemos definir $\tilde{\delta}(\alpha)=\delta(\alpha)$. Pela Afirmação 1 acima temos que $\tilde{\delta}(\alpha)=\lambda_{\alpha} \alpha$ para algum $\lambda_{\alpha} \in k$. Observamos que este argumento prova também o caso em que $n=1$.

Vamos supor agora que $x$ não é projetivo e suponha por hipótese de indução que $\tilde{\delta}(\beta)$ está definido para todo $\beta$ tal que $e(\beta)=y \operatorname{com} n(e(\beta))<n, n>1$.

Neste caso existe a sequência de translação $\tau x \longrightarrow \oplus_{i=1}^{s} y_{i} \longrightarrow x$ em $\Delta$ terminando em $x$ e denotemos por $\alpha_{i}: \tau x \rightarrow y_{i}$ e $\beta_{i}: y_{i} \rightarrow x$ as flechas correspondentes para $i=1, \ldots, s$. Por indução temos que para cada $i, \tilde{\delta}\left(\alpha_{i}\right)$ está definida, digamos $\tilde{\delta}\left(\alpha_{i}\right)=$ $\lambda_{i} \alpha_{i}$ para $\lambda_{i} \in k$. E assim podemos definir $\tilde{\delta}\left(\beta_{j}\right)=\sum_{i \neq j} \lambda_{i} \beta_{j}$. Desta maneira, temos que $\tilde{\delta}$ assim definida é a uma derivação normalizada em $\Lambda$ e que estende $\delta$.

Agora suponhamos que $\delta \in \operatorname{Der}^{n}(\Lambda)$ é tal que $\psi(\delta)$ é interno. Nós afirmamos que $\delta \in \operatorname{Der}^{n, 0}(\Lambda)$.

Para isto é suficiente mostrar que se

$$
\tau x \stackrel{\alpha_{i}}{\longrightarrow} \sum_{i=1}^{s} y_{i} \stackrel{\beta_{i}}{\longrightarrow} x
$$

é uma sequência de translação tal que $\delta\left(\alpha_{i}\right)=0 \forall i=1, \ldots, s$, então existe $\mu \in k$ tal que $\left(\delta-\delta_{\mu_{e x}}\right)\left(\beta_{i}\right)=0 \forall i=1, \ldots, s$. Seja $\delta\left(\beta_{i}\right)=\lambda_{i} \beta_{i} \forall i=1, \ldots, s$. Como $0=$ $\delta\left(\sum_{i=1}^{s} \beta_{i} \alpha_{i}\right)=\sum_{i=1}^{s} \delta\left(\beta_{i} \alpha_{i}\right)=\sum_{i=1}^{s} \delta\left(\beta_{i}\right) \alpha_{i}+\sum_{i=1}^{s} \beta_{i} \delta\left(\alpha_{i}\right)$ temos $\sum_{i=1}^{s} \lambda_{i} \beta_{i} \alpha_{i}=0$. Por outro lado, $\sum_{i=1}^{s} \beta_{i} \alpha_{i}=0$. Desta maneira, temos $\lambda_{1}=\lambda_{2}=\ldots, \lambda_{s}=\lambda$. Então vamos escolher $\mu=\lambda$.

Logo, $\left(\delta-\delta_{\mu_{e_{x}}}\right)\left(\beta_{i}\right)=\delta\left(\beta_{j}\right)-\delta_{\mu_{x}}\left(\beta_{j}\right)=\lambda \beta_{j}-\left(\beta_{j} \mu_{e_{x}}-\mu_{e_{x}} \beta_{j}\right)=0$ para $1 \leq j \leq r$.

Seja $\delta \in \operatorname{Der}^{n, 0}(\Lambda)$. Então é fácil ver que $\psi(\delta) \in \operatorname{Der}^{n, 0}(k \mathcal{O}(\Delta))$. De fato, seja $\delta=\delta_{\lambda}$, onde $\lambda=\sum_{i=1}^{n} \mu_{i} e_{x} \in \Lambda$.

Seja $j$ um vértice de $\mathcal{O}(\Delta)$. Sejam $\alpha_{1}, \ldots, \alpha_{r}$ (respectivamente $\beta_{1}, \ldots, \beta_{s}$ ) as flechas de $\mathcal{O}(\Delta)$ começando (respectivamente terminando) em $j$. Sejam $x_{1}, x_{2}, \ldots, x_{r}$ os vértices que estão em $\left(x_{j}\right)^{+}$em $\Delta$ e $y_{1}, y_{2}, \ldots, y_{s}$ os vértices que estão em $\left(x_{j}\right)^{-}$. Seja $\nu_{j}=\sum_{i=1}^{r} \mu_{i j} e_{j}+\sum_{i=1}^{s} \mu_{j i} e_{j}$. Então $\psi(\delta)=\sum_{j} \delta_{\nu_{j}} \in \operatorname{Der}^{n, 0}(k \mathcal{O}(\Delta))$. 


\subsection{Ciclos e contornos para álgebras de tipo mesh}

Nesta seção vamos tomar uma álgebra de tipo mesh $\Lambda=\frac{k \Delta}{\mu}$ com a seguinte propriedade: o grafo-órbita $\overline{\mathcal{O}}\left(\Delta^{\prime}\right)$ de $\Delta^{\prime}$ é árvore para toda subaljava $\Delta^{\prime} \subseteq \Delta$. Então neste caso, poderemos descrever o que ocorre com os ciclos chamados irredutíveis em $(\Delta, \mu)$. Iremos inicialmente introduzir os conceitos de contornos, ciclos, redutibilidade e contratilidade.

Em [7], Assem e Liu obtiveram algumas caracterizações e técnicas de construção de álgebras fortemente simplesmente conexas. O seguinte resultado é um critério que nos permite verificar quando uma $k$-categoria localmente limitada [16] é fortemente simplesmente conexa:

Teorema 3.5. ( [7]): Seja $\Lambda$ uma k-categoria localmente limitada triangular conexa. Então as seguintes condições são equivalentes:

(a) $\Lambda$ é fortemente simplesmente conexa.

(b) Para toda apresentação $\Lambda \cong \frac{k \Delta_{\Lambda}}{I_{\Lambda}}$, o grupo fundamental de toda subaljava convexa plena conexa de $\left(\Delta_{\Lambda}, I_{\Lambda}\right)$ é trivial.

(c) Para toda apresentação $\Lambda \cong \frac{k \Delta_{\Lambda}}{I_{\Lambda}}$, todo ciclo irredutivel em $\Delta_{\Lambda}$ é um contorno irredutivel e todo contorno irredutivel é naturalmente contrátil.

(d) Existe uma apresentação $\Lambda=\frac{k \Delta_{\Lambda}}{I_{\Delta}}$ tal que todo ciclo irredutível em $\Delta_{\Lambda}$ é um contorno irredutivel, e todo contorno irredutivel é naturalmente contrátil.

Seja $\Delta$ uma aljava localmente finita e dirigida.

Definição 3.6. Um contorno $C_{x y}=(p, q)$ em $\Delta$ de $x$ para y é um par de caminhos $p, q$ de comprimento positivo tendo a mesma fonte $x$ e o mesmo poço $y$.

Definição 3.7. Seja $C_{x y}=(p, q)$ um contorno com $p=\alpha_{n} \ldots \alpha_{2} \alpha_{1}$ eq $q=\beta_{m} \ldots \beta_{2} \beta_{1}$. Dizemos que $C_{x y}$ é um contorno próprio sempre que $\alpha_{1} \neq \beta_{1}$ e $\alpha_{n} \neq \beta_{m}$. 
Um contorno $(p, q)$ em $\Delta$ de $x$ para $y$ é chamado interlaçado se os caminhos $p$ e $q$ têm outro ponto em comum diferente de $x$ e $y$. Um contorno $(p, q)$ é chamado contorno redutível se existirem caminhos $p=p_{0}, p_{1}, \ldots, p_{m}=q$ em $\Delta$ de $x$ para $y$ tais que, para cada $1 \leq i \leq m$, o contorno $\left(p_{i-1}, p_{i}\right)$ seja interlaçado. (Neste caso, nós dizemos que $p$ é redutível a $q$ ). Caso contrário, ele é dito irredutível.

Algumas observações:

1. Todo contorno $(p, q)$ interlaçado é redutível, mas a recíproca nem sempre é verdadeira. Basta vermos o exemplo 2.11. Ao considerarmos o contorno $C_{1,6}=$ $\left(p=\alpha_{5} \alpha_{2} \alpha_{1}, q=\alpha_{7} \alpha_{6} \alpha_{3}\right)$. Temos que $p$ e $q$ não são interlaçados, mas existe o caminho $p_{1}=\alpha_{5} \alpha_{4} \alpha_{3}$ de 1 para 6 de maneira que $\left(p_{0}=p, p_{1}\right)$ é interlaçado e $\left(p_{1}, p_{2}=q\right)$ é interlaçado. Logo, $C_{1,6}$ é um contorno redutível.

2. Um contorno irredutível é sempre um contorno próprio.

3. contorno $(p, q)$ onde $p$ é uma flecha é um contorno irredutível. De fato, suponha que $C_{x y}=(p, q)$ é um contorno redutivel. Neste caso, deve existir um caminho $p_{1}$ que é interlaçado com $p_{0}=p$, ou seja, $p_{1}$ deve ter um vértice diferente de $x$ e $y$ em comum com $p$. Mas como $p$ é uma flecha de $x$ para $y$, isto não pode ocorrer.

Vamos também definir uma ordem parcial nos contornos em $\Delta$ como segue. Sejam $\left(p_{1}, q_{1}\right)$ e $\left(p_{2}, q_{2}\right)$ dois contornos de $x_{1}$ para $y_{1}$ e $x_{2}$ para $y_{2}$, respectivamente. Então $\left(p_{1}, q_{1}\right) \leq\left(p_{2}, q_{2}\right)$ se ou $\left(p_{1}, q_{1}\right)=\left(p_{2}, q_{2}\right)$ ou $\left(x_{1}, y_{1}\right) \neq\left(x_{2}, y_{2}\right)$, e então $x_{1}$ é um sucessor de $x_{2}$, e $y_{1}$ é um predecessor de $y_{2}$. 
No seguinte exemplo, nós temos $\left(p_{1}, q_{1}\right) \leq\left(p_{2}, q_{2}\right)$ :

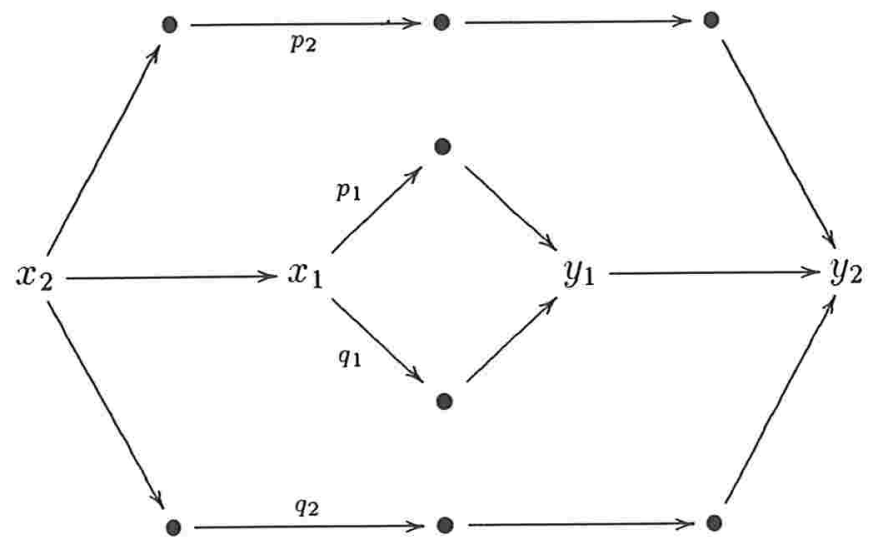

Precisamos de uma noção de contratilidade de contornos. Seja uma aljava como antes, ou seja, localmente finita e dirigida, e $I$ um ideal admissível de $k \Delta$. Dois caminhos $p$ e $q$ de $x$ para $y$ em $\Delta$ são chamados naturalmente homotópicos em $(\Delta, I)$ se existir uma sequência de caminhos $p=p_{0}, p_{1}, \ldots, p_{m}=q$ em $\Delta$ tal que, para cada $0 \leq i<m, p_{i}$ e $p_{i+1}$ têm subcaminhos $q_{i}$ e $q_{i+1}$, respectivamente, que estão envolvidos na mesma relação minimal em $(\Delta, I)$. Um contorno $(p, q)$ é chamado naturalmente contrátil se os caminhos $p, q$ são naturalmente homotópicos em $(\Delta, I)$.

É interessante obsevar que os caminhos $p$ e $q$ de um contorno podem ser homotópicos mas não serem naturalmente homotópicos. Vejamos o exemplo abaixo:

Exemplo 3.8. Seja a aljava $\Delta$ :

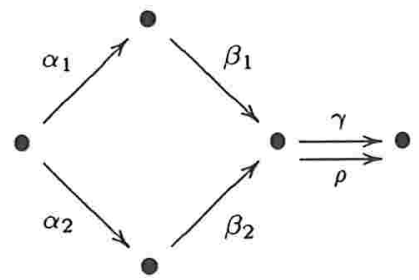

e $I$ o ideal gerado por $\beta_{1} \alpha_{1}-\beta_{2} \alpha_{2}, \gamma \beta_{1} \alpha_{1}-\rho \beta_{1} \alpha_{1}$.

Os caminhos $\beta_{1} \alpha_{1}, \beta_{2} \alpha_{2}$ são naturalmente homotópicos em $(\Delta, I)$, então o contorno $\left(\beta_{1} \alpha_{1}, \beta_{2} \alpha_{2}\right)$ é naturalmente contrátil.

Mas, os caminhos $\gamma$ e $\rho$ são homotópicos em $(\Delta, I)$, mas são naturalmente homotópicos, então o contorno $(\gamma, \rho)$ não é naturalmente contrátil. 
Proposição 3.9. Seja $\Delta$ uma aljava finita, dirigida e com translação e $\Lambda=\frac{k \Delta}{\mu} a$ álgebra de tipo mesh associada a $\Delta$. Então todo contorno irredutível naturalmente contrátil em $(\Delta, \mu)$ é do tipo

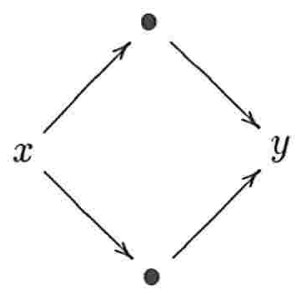

com $x=\tau y$.

Prova: Suponhamos que exista um contorno irredutível $C_{x y}=(p, q)$ naturalmente contrátil em $(\Delta, \mu)$ que não seja do tipo acima. Suponhamos que $x \neq \tau y$. Como $C_{x y}$ é naturalmente contrátil, $p$ e $q$ são naturalmente homotópicos, portanto existe uma sequência de caminhos $p=p_{0}, p_{1}, \ldots, p_{m}=q$ em $(\Delta, \mu)$ onde $p_{i}$ e $p_{i-1}$, $0 \leq i<m$ têm subcaminhos $q_{i}$ e $q_{i-1}$ que estão envolvidos na mesma relação mesh minimal em $(\Delta, \mu)$. Então cada par $\left(q_{i-1}, q_{i}\right)$ tem a mesma fonte $x^{\prime}=\tau y^{\prime}$ e o mesmo poço $y^{\prime}$ em comum e como estamos supondo $x \neq \tau y$, pelo menos um deles, ou $x^{\prime}$ ou $y^{\prime}$ deve ser distinto de $x$ ou $y$. Disto teremos que os caminhos $p_{i}$ e $p_{i-1}$ com $0 \leq i<m$ serão interlaçados. Mas isto é uma contradição pois $C_{x y}$ é irredutível. Então em particular $y$ é não projetivo e $x=\tau y$. Logo, temos

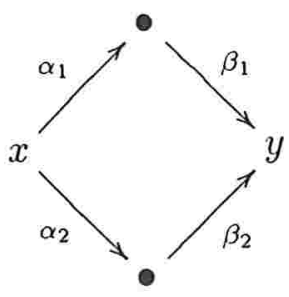

Falta ainda mostrarmos que $p=\beta_{1} \alpha_{1}$ e $q=\beta_{2} \alpha_{2}$.

Suponhamos que $p \neq \beta_{1} \alpha_{1}$ e escrevemos $p=\gamma_{n} \gamma_{n-1} \ldots \gamma_{1}$. Claramente, o contorno $(p, q)$ não poderá ser contrátil pois nem $\gamma_{1}$ e nem $\gamma_{n}$ podem pertencer a subcaminhos de $p$ que estejam em alguma relação mesh minimal.

De modo similar vemos que $q$ deve ser igual a $\beta_{2} \alpha_{2}$. 
Definição 3.10. Uma subaljava $C$ de $\Delta$ é um ciclo se cada vértice em $C$ pertencer a exatamente duas flechas em $C$ e existir uma enumeração $\left\{x_{0}, x_{1}, \ldots, x_{n-1}, x_{n}=x_{0}\right\}$ dos vértices de $C$ tal que existe uma aresta entre $x_{i-1}$ e $x_{i}$ em $C$, para todo $1 \leq i \leq n$.

Seja $C$ um ciclo que não é um contorno. Vamos denotar por $n f(C)$ o número de fontes de $C$ (que pela definição de ciclo é igual ao número de poços de $C n p(C)$ ). Então $n f(C)>1$. O ciclo $C$ é chamado ciclo redutível se existirem dois vértices $x, y$ em $C$, e um caminho $p: x \rightarrow \ldots \rightarrow y$ em $\Delta$ como segue:

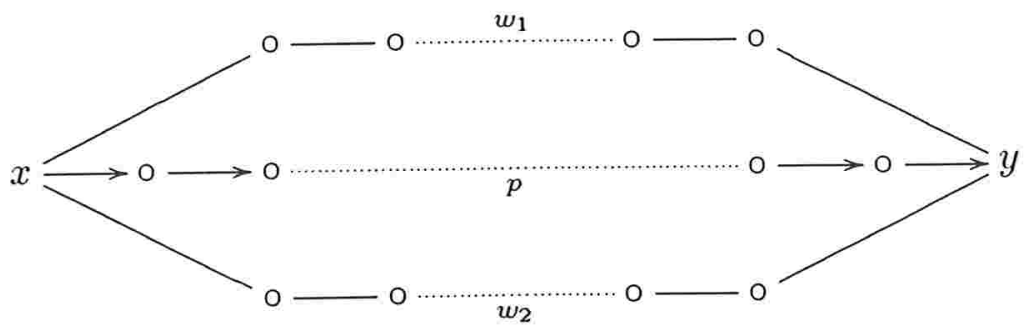

onde o ciclo $C$ consiste dos passeios $w_{1}$ e $w_{2}$, tais que ambos $p^{-1} w_{1}$ e $p^{-1} w_{2}$ são ciclos e $n f\left(p^{-1} w_{1}\right)<n f(C), n f\left(p^{-1} w_{2}\right)<n f(C)$. Nós então dizemos que um caminho tal como $p$ reduz o ciclo $C$. Um ciclo $C$ é chamado irredutível se ele é um contorno irredutível, ou se ele não é um contorno, mas não é redutível no sentido acima.

Inicialmente, temos o seguinte Lema que trata de uma propriedade dos ciclos irredutíveis que não são contornos irredutíveis.

Lema 3.11. Sejam $\Delta$ uma aljava dirigida e localmente finita e w um ciclo irredutivel em $\Delta$ que não seja um contorno irredutível, isto é, w é do tipo: 


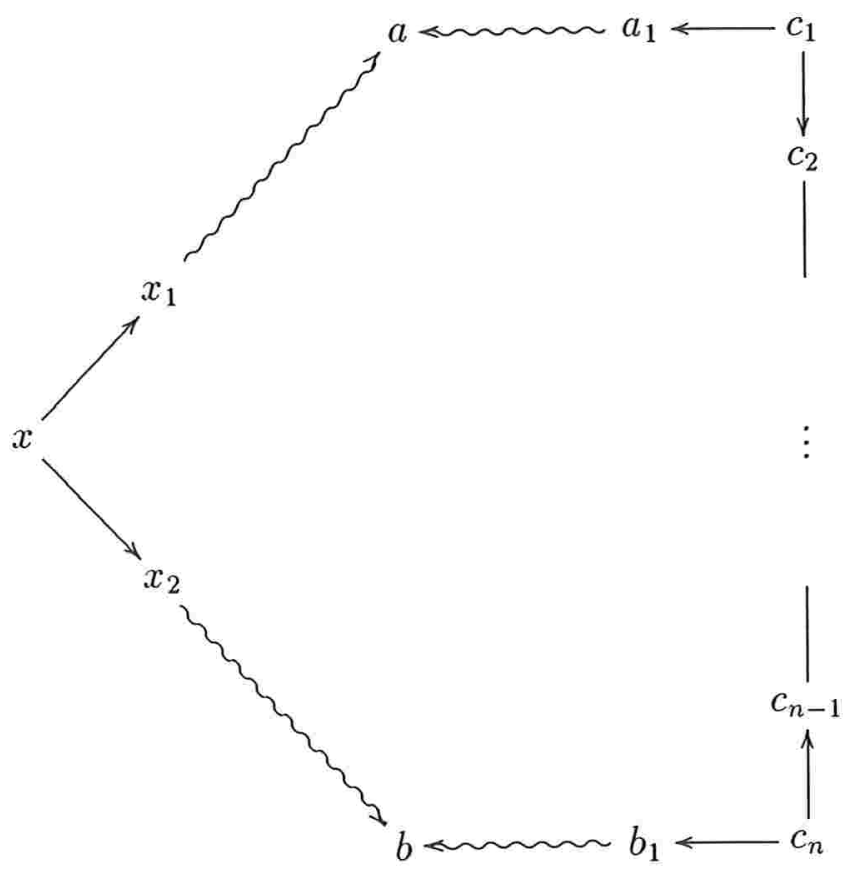

onde $x, c_{1}$ e $c_{n}$ são fontes de $w$ e podem coincidir. A notação é de um caminho orientado em $w$. Denotamos por $v: c_{1} \longrightarrow c_{2}-\cdots-c_{n-1} \longleftarrow c_{n}$ o passeio em $\Delta$ ligando $c_{1}$ e $c_{n}$ e não passando por $x$. Então, para cada $1 \leq i \leq n$, não existe nenhum caminho de $x$ para $c_{i}$ e nem caminho de $c_{i}$ para $x$.

\section{Prova:}

Vamos supor que para algum $1 \leq i \leq n$ existe um caminho de $x$ para $c_{i}$ ou existe um caminho de $c_{i}$ para $x$. Denotamos por $\nu$ e $\xi$ os dois passeios distintos de $w$ ligando $x$ e $c_{i}$ passando por $a$ e $b$ respectivamente. Vamos escrever $v=v_{2} v_{1}$ onde $c_{i}$ é o vértice em comum de $v_{1}$ e $v_{2}$.

Suponhamos inicialmente que existe um caminho $\theta$ de $x$ para $c_{i}$. Neste caso, iremos ter dois ciclos: $w_{1}=\theta^{-1} \nu$ e $w_{2}=\theta^{-1} \xi$. Vamos mostrar que $n f\left(w_{i}\right)<n f(w) \forall i=1,2$, obtendo uma contradição ao fato de $w$ ser irredutível.

- Se $c_{i}$ é um poço em $w$ observamos que $n f(w)=1+n f\left(v_{1}\right)+n f\left(v_{2}\right)$, logo $n f\left(w_{i}\right)=n f\left(v_{i}\right)<n f(w)$ para $i=1,2$. 
- Se $c_{i}$ não é um poço em $w$ temos que $n f(w)=n f\left(v_{1}\right)+n f\left(v_{2}\right)$ e como $n f\left(v_{i}\right) \geq 1$, temos $n f\left(w_{i}\right)=n f\left(v_{i}\right)<n f(w)$ para $i=1,2$.

No caso que existe um caminho $\theta$ de $c_{i}$ para $x$ temos novamente $w_{1}=\theta^{-1} \nu$ e $w_{2}=\theta^{-1} \xi$ os ciclos induzidos por $\theta$ e o argumento é muito similar ao anterior.

- Se $c_{i}$ é fonte em $w$ observamos que $n p(w)=n p\left(v_{1}\right)+n p\left(v_{2}\right)+2$, e então, $n p\left(w_{i}\right)=n p\left(v_{i}\right)+1<n p(w)$ para $i=1,2$.

- Se $c_{i}$ não é fonte em $w$ temos que $n p(w)=n p\left(v_{1}\right)+n p\left(v_{2}\right)+1$, e então $n p\left(w_{i}\right)=$ $n p\left(v_{i}\right)+1<n p(w)$ pois $n p\left(v_{i}\right) \geq 1$ para $i=1,2$. Obtemos então nos dois casos anteriores uma contradição ao fato de $w$ ser irredutivel.

E então concluímos que se existe caminho $\theta$ de algum $c_{i}$ para $x$ ou de $x$ para algum $c_{i}$, este caminho reduzirá o ciclo $w$ e isto não pode ocorrer.

Teorema 3.12. Seja $\Delta$ uma aljava conexa, finita, dirigida e com translação. Se $\overline{\mathcal{O}}\left(\Delta^{\prime}\right)$ é árvore para toda subaljava $\Delta^{\prime} \subseteq \Delta$ do tipo $\tilde{\mathbf{A}}_{n}$, então todo ciclo irredutível em $\Delta$ é um contorno irredutivel.

\section{Prova:}

Suponhamos que exista um ciclo irredutível $w$ em $\Delta$ que não seja um contorno irredutível. Então $w$ é da forma como descrevemos no Lema anterior. Vamos considerar a mesma notação usada acima para o ciclo $w$ e também denominamos os seguintes caminhos em $w$ :

$p: x \longrightarrow \cdots \longrightarrow a, p_{1}: a \longleftarrow \cdots \longleftarrow a_{1} \longleftarrow c_{1}$,

$q: x \longrightarrow \cdots \longrightarrow b, q_{1}: b \longleftarrow \cdots \longleftarrow b_{1} \longleftarrow c_{n}$.

Consideramos os passeios $w_{1}=q p^{-1}$ e $w_{2}=q_{1} v p_{1}^{-1}$

Observamos primeiro que $o(a) \neq o(b)$. De fato, suponha que $a=\tau^{s} b$ e sem perda de generalidade assumimos que $s>0(a \neq b$ pela definição de ciclo). Então deve existir um caminho $\theta$ de $a$ para $b$ e neste caso, $\theta$ reduz $w$, o que é uma contradição. 
Afirmação : Se o passeio $w_{2}$ passa através de $y=\tau^{s} x$, para algum $s \neq 0$, então $s<0$ e $y$ pertence a $p_{1}$ ou a $q_{1}$.

Primeiro observamos que $y \neq c_{i}$ para todo $i=1, \ldots, n$ pois caso contrário teremos um caminho entre $c_{i}$ e $x$ e isto contradiz o Lema anterior.

Suponhamos que $s>0$. Então $y$ não pode pertencer a $p_{1}$ pois senão existiria um caminho de $c_{1}$ para $x$, o que seria uma contradição ao Lema anterior. Analogamente temos que $y$ não pode pertencer a $q_{1}$. Então $s<0$ e $y$ pertence a $p_{1}$ ou a $q_{1} \mathrm{e}$ temos a nossa Afirmação provada.

Vamos agora escrever $p_{1}$ e $q_{1}$ da seguinte maneira:

$p_{1}: c_{1}=y_{1} \longrightarrow \cdots \longrightarrow y_{r}=a \mathrm{e}$

$q_{1}: c_{n}=y_{1}^{\prime} \longrightarrow \cdots \longrightarrow y_{s}^{\prime}=b$.

Escolhemos um vértice $z$ da seguinte maneira: se o caminho $p_{1}$ cruza a $\tau$-órbita de $x$, escolhemos $j$ o menor índice tal que $y_{j}$ esteja na $\tau$-órbita e tomamos $z=y_{j}$. Se $p_{1}$ não cruza a $\tau$-órbita de $x$, tomamos $z=a$.

Analogamente, escolhemos um vértice $z^{\prime}$ da seguinte maneira: se o caminho $q_{1}$ cruza a $\tau$-órbita de $x$, escolhemos $l$ o menor índice tal que $y_{l}^{\prime}$ esteja na $\tau$-órbita de $x$ e tomamos $z^{\prime}=y_{l}^{\prime}$. Se $q_{1}$ não cruza a $\tau$-órbita de $x$, tomamos $z^{\prime}=b$.

Assim, temos que existem os seguintes caminhos:

(i) um caminho $\bar{p}$ de $x$ para $z$ (que poderá ser $p$ no caso em que $z=a$ );

(ii) um caminho $\bar{p}_{1}$ de $c_{1}$ para $z$;

(iii) um caminho $\bar{q}$ de $x$ para $z^{\prime}$; e

(iv) um caminho $\bar{q}_{1}$ de $c_{n}$ para $z^{\prime}$.

Logo, obtemos o seguinte ciclo: 


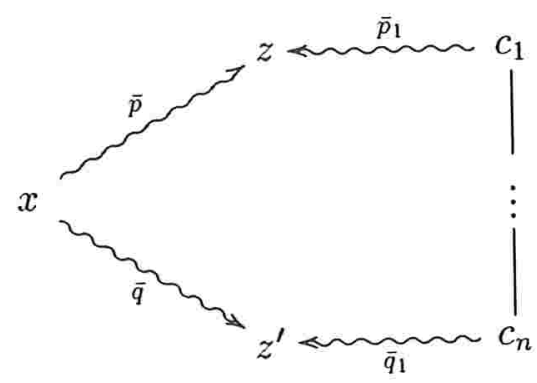

Denominamos $\bar{w}_{1}=\bar{q} \bar{p}^{-1}$ e $\bar{w}_{2}=\bar{q}_{1} v \bar{p}_{1}^{-1}$.

Observe que $\bar{w}_{1}$ e $\bar{w}_{2}$ induzem dois caminhos diferentes em $\overline{\mathcal{O}}(\Delta)$ entre $o(z)$ e $o\left(z^{\prime}\right)$ (pois um caminho passa pela órbita de $x$ e o outro não) e isto contradiz a nossa hipótese de indução.

Teorema 3.13. Seja $\Delta$ uma aljava dirigida, conexa, finita, com translação e vamos assumir que $\overline{\mathcal{O}}\left(\Delta^{\prime}\right)$ seja árvore para toda subaljava $\Delta^{\prime}$ de $\Delta$ de tipo $\tilde{\mathbf{A}}_{n}$. Se $C_{x y}$ é um contorno irredutivel em $\Delta$ então $y=\tau^{-1} x$ e o contorno $C_{x y}$ é do tipo

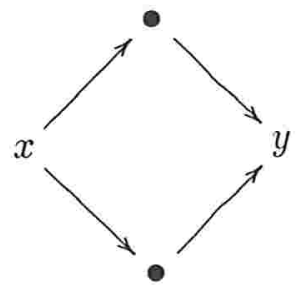

\section{Prova:}

Afirmação: O contorno $C_{x y}$ deve cruzar um vértice $z=\tau^{s} x, s<0$.

De fato, suponhamos que $C_{x y}$ não cruze nenhum vértice $z=\tau^{s} x \operatorname{com} s \neq 0$ e seja 
$C_{x y}$ como abaixo:

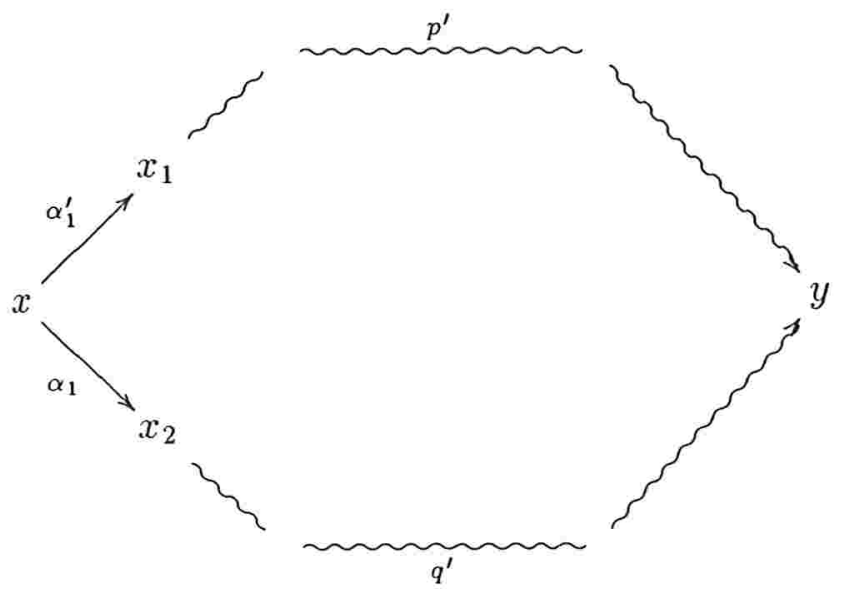

onde $C_{x y}=(p, q) \operatorname{com} p=p^{\prime} \alpha_{1}^{\prime}$ e $q=q^{\prime} \alpha_{1}$.

Como o contorno $C_{x y}$ é irredutível, então ele é próprio, logo teremos que $x_{1} \neq x_{2}$ (e aqui $o\left(x_{1}\right) \neq o\left(x_{2}\right)$ pois senão $C_{x y}$ seria redutível). Então o passeio $x_{1} \longleftarrow x \longrightarrow x_{2}$ em $C_{x y}$ que cruza a $\tau$-órbita de $x$ e o passeio $x_{1} \sim p^{\prime} \sim y \ll q^{\prime} \sim x_{2}$ em $C_{x y}$ que não cruza a $\tau$-órbita de $x$ induzem dois passeios diferentes entre $o\left(x_{1}\right)$ e $o\left(x_{2}\right)$ pois um passa pela $\tau$-órbita de $x$ e outro não. Logo, obtemos o seguinte passeio em $\overline{\mathcal{O}}\left(C_{x y}\right)$ :

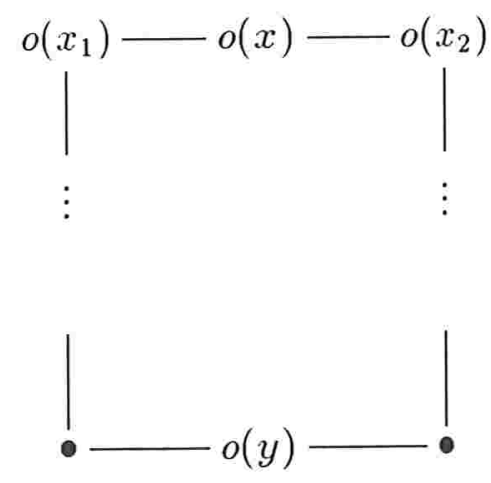

o que é uma contradição ao fato de $\overline{\mathcal{O}}\left(C_{x y}\right)$ ser árvore. Portanto temos que $C_{x y}$ cruza um vértice $z=\tau^{s} x$ para algum $s$. Como $\Delta$ não possui circuitos orientados concluímos que $s<0$ e a afirmação está provada. Em particular, $x$ não é um vértice injetivo. Vamos mostrar agora que $y=\tau^{-1} x$.

Sabemos que $z=\tau^{s} x, s<0$ um vértice em $p$ ou em $q$. Suponhamos por 
contradição que $y \neq \tau^{-1} x$.

Sabemos que existem as flechas $x_{1} \stackrel{\beta_{1}^{\prime}}{\longrightarrow} \tau^{-1} x$ e $x_{2} \stackrel{\beta_{1}}{\longrightarrow} \tau^{-1} x$ chegando em $\tau^{-1} x$ e observamos também que existe o caminho $\varphi: \tau^{-1} x_{1} \rightarrow \ldots \rightarrow \tau^{s} x_{1}=z$. Sendo $\varphi^{\prime}$ um caminho de $C_{x y}$ que vai de $z$ a $y$, temos que o caminho $p_{1}: \varphi^{\prime} \varphi \beta_{1}^{\prime} \alpha_{1}^{\prime}$ de $x$ para $y$ é interlaçado com $p$ e com $p_{2}: \varphi^{\prime} \varphi \beta_{1} \alpha_{1}$ que é interlaçado com $q$ e neste caso o contorno $C_{x y}$ é redutível e isto é uma contradição.

Queremos mostrar com as notações acima que $p=\beta_{1}^{\prime} \alpha_{1}^{\prime}$ e $q=\beta_{1} \alpha_{1}$. Suponhamos, por exemplo que $p \neq \beta_{1}^{\prime} \alpha_{1}^{\prime}$. Então existe um vértice $y_{1} \neq x_{1}$ e uma flecha $\beta_{2}^{\prime}: y_{1} \rightarrow y$ tal que $p=\beta_{2}^{\prime} w \alpha_{1}^{\prime}$ onde $w$ é um caminho de $x_{1}$ para $y_{1}$. Claramente, também existe uma flecha $\alpha_{2}^{\prime}: x \rightarrow y_{1}$.

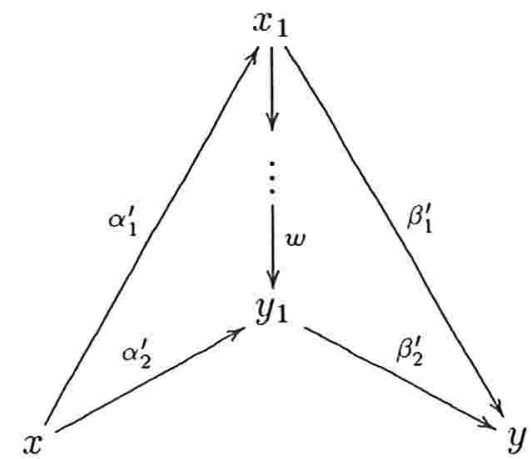

Observamos que nenhum vértice do caminho $w$ cruza a $\tau$-órbita de $x$ e também, como $x_{1} \neq y_{1}$ temos que $o\left(x_{1}\right) \neq o\left(y_{1}\right)$ pois senão em ambos os casos teríamos circuito orientado em $\Delta$. Disto, obtemos para a subaljava $\Delta_{1}$ :

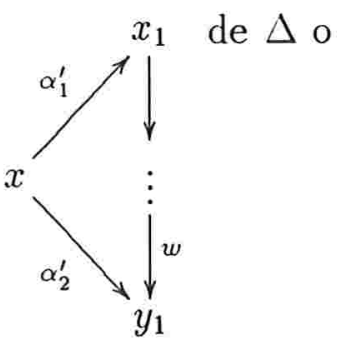

seguinte grafo-órbita:

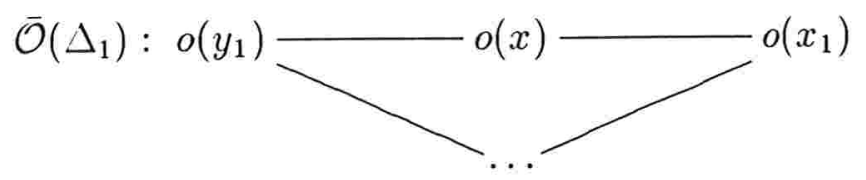


onde temos dois passeios distintos entre $o\left(x_{1}\right)$ e $o\left(y_{1}\right)$ pois um deles passa pela $\tau$ órbita de $x$ e outro não e isto contradiz a hipótese de que $\overline{\mathcal{O}}(\Delta)$ é uma árvore.

Similarmente, $q$ deve ser igual a $\beta_{1} \alpha_{1}$, e temos o que queríamos.

\subsection{Demonstração do resultado principal}

Vejamos agora as equivalências do Teorema 3.1 que faltam ser verificadas.

A implicação $(\mathrm{a}) \Rightarrow(\mathrm{d})$ vale pelo Teorema 3.5 de Assem e Liu juntamente com a Proposição 3.9 .

Para mostrarmos $(\mathrm{b}) \Rightarrow(\mathrm{e})$ basta vermos que como $H^{1}(\Lambda)=0$ temos pelo Teorema 3.2 e pela hipótese que $\Delta$ não possui flechas múltiplas que $\Delta$ é $\tilde{A}_{1, p}$-livre, então pelo Teorema 3.4 temos que $H^{1}(k \mathcal{O}(\Delta))=0$; logo, por [32] temos que $\mathcal{O}(\Delta)$ é árvore. Por outro lado, para obtermos a implicação (e) $\Rightarrow(\mathrm{b})$ basta vermos que como $\mathcal{O}(\Delta)$ é árvore, temos que $H^{1}(k \mathcal{O}(\Delta))=0$ por [32] e que também vale o Teo.3.4. Logo, $0=H^{1}(k \mathcal{O}(\Delta)) \cong H^{1}(\Lambda)$.

(f) $\Rightarrow(\mathrm{d})$

Primeiro vemos pelo Teorema 3.12 que (f) implica que todo ciclo irredutível é um contorno irredutível e pelo Teorema 3.13 que todo contorno irredutivel é do tipo $C_{x y}=\quad \operatorname{com} \tau^{-1} x=y$. Neste caso temos que $C_{x y}$ obviamente é

naturalmente contrátil.

(e) $\Rightarrow$ (f)

Suponhamos que a aljava-órbita $\mathcal{O}(\Delta)$ de $\Delta$ seja árvore. Consideremos o grafo-órbita $\overline{\mathcal{O}}(\Delta)$. Basta observarmos que se $\Delta^{\prime}$ é subaljava de $\Delta$ então $\overline{\mathcal{O}}\left(\Delta^{\prime}\right)$ é um subgrafo de $\overline{\mathcal{O}}(\Delta)$. Logo, como $\overline{\mathcal{O}}(\Delta)$ é árvore, o seu subgrafo $\overline{\mathcal{O}}\left(\Delta^{\prime}\right)$ também é árvore para toda $\Delta^{\prime}$ subaljava de $\Delta$. 


\subsection{Consequências e exemplos}

Com o Teorema 3.1 obtemos uma caracterização das álgebras de tipo mesh que são fortemente simplesmente conexas. Temos também que as álgebras de tipo mesh que satisfazem o Teorema 3.1 possuem a condição de separação e portanto possuem uma componente pós-projetiva (ver [17]). Logo, podemos enunciar o seguinte Corolário:

Corolário 3.14. Seja $\Lambda$ uma aljava finita, dirigida e com translação e $\Lambda=\frac{k \Delta}{\mu}$ a álgebra de tipo mesh associada a $\Delta$. Se $\Lambda$ satisfaz alguma das propriedades do Teorema 3.1 então $\Lambda$ possui componente pós-projetiva.

Observamos que existem álgebras de tipo mesh que possuem componentes pós-projetivas, porém, não satisfazem as propriedades do Teorema 3.1.

Exemplo 3.15. Vamos considerar a álgebra $\Lambda=\frac{k \Delta}{\mu}$ onde

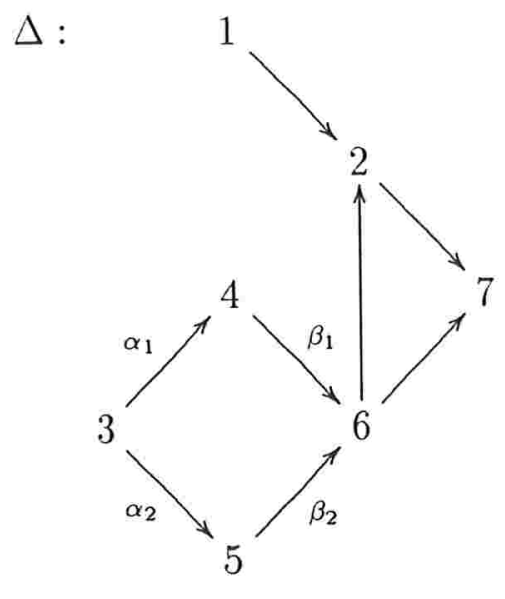

e $\mu=<\beta_{1} \alpha_{1}-\beta_{2} \alpha_{2}>$.

Claramente esta álgebra não satisfaz a propriedade (d) do Teorema 3.1 , pois ela possui o contorno irredutível $C_{6,7}$ que não é naturalmente contrátil em $(\Delta, \mu)$. 
Para sabermos que esta álgebra possui componente pós-projetiva usamos o procedimento de Dräxler e de la Peña (ver o Teorema 1.26 no Capítulo 1). A álgebra $\Lambda$ pode ser vista como a extensão por um ponto $\Lambda=B[M]$ onde $M=\operatorname{rad} P_{7}$ e $B=\frac{k \Delta^{\prime}}{\mu}$ onde

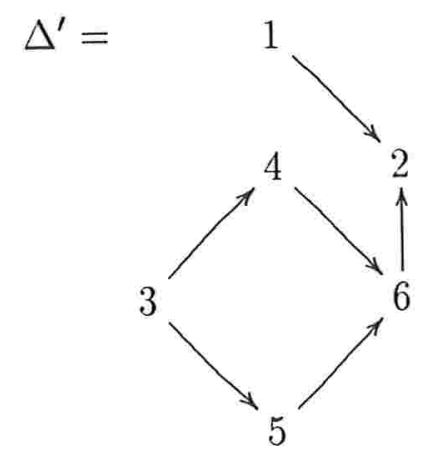

Como a álgebra $B$ possui componente pós-projetiva, basta termos que $\operatorname{rad} P_{7}=P_{2} \oplus$ $P_{6}$ seja dirigido com $B$-módulo e isto ocorre (podemos construir a aljava de AuslanderReiten de $B$ e verificar que não existe um $B$-módulo indecomponível não projetivo $W$ tal que $P_{2} \preceq \tau W$ e $W \preceq P_{6}$ ou $P_{6} \preceq \tau W$ e $\left.W \preceq P_{2}\right)$.

De fato, a aljava de Auslander-Reiten $\Gamma_{B}$ de $B$ é a seguinte:

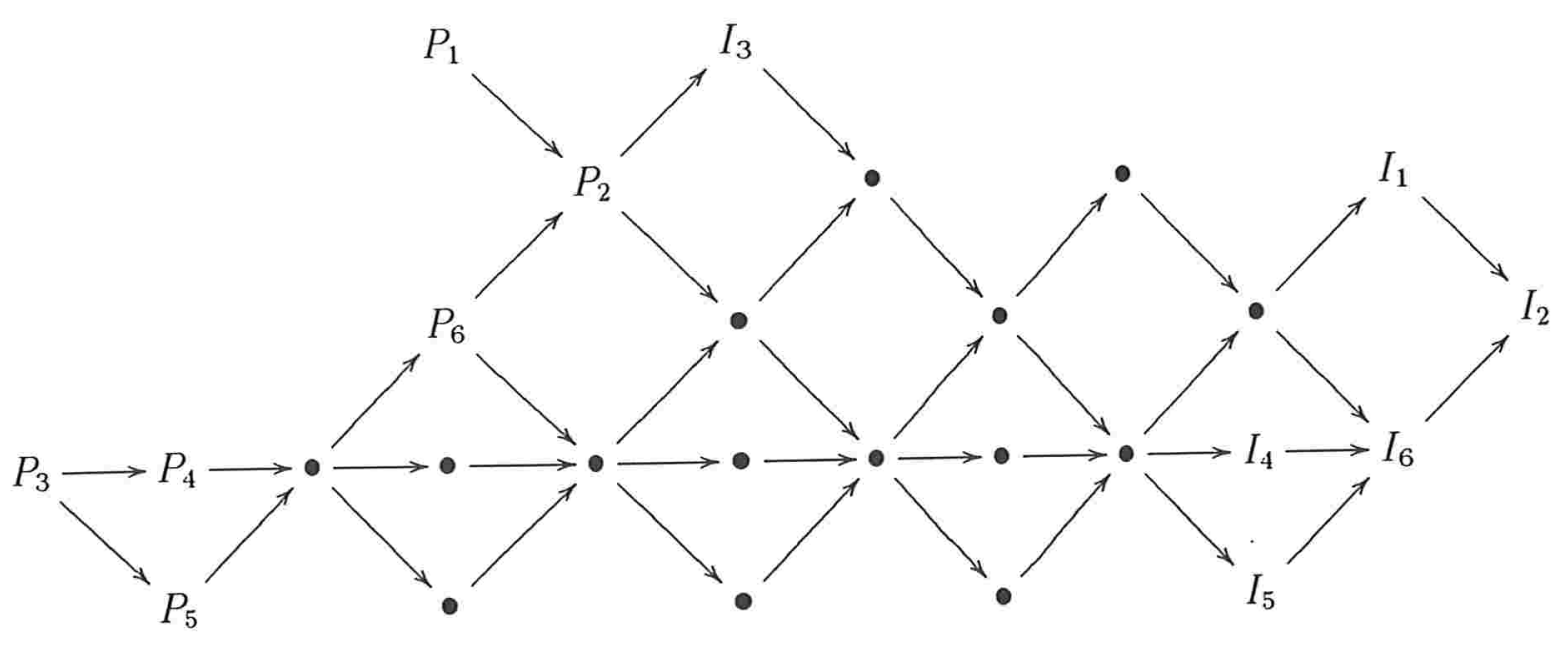




\section{Referências Bibliográficas}

[1] L. Algeleri-Hügel, F. U. Coelho, Postprojective components for algebras in $\mathcal{H}_{1}$, Arch. Math. 69 (1997), 1-7.

[2] I. AssEm, J. A. DE LA PEÑA, The fundamental groups of a triangular algebra, Comm. in Algebra, Vol.24(1) (1996), 187-208.

[3] I. Assem, A. Skowroński, On Some Classes of Simply Connected Algebras, Proc. London Math. Soc. (3)56 (1988), 417-450.

[4] I. Ássem, A. Skowrońki, On tame repetitive algebras, Fund. Math. 142 (1993), 59-84.

[5] I. Assem, P.Brown, Strongly Simply Connected Auslander Algebras, Glasgow Math. J. 39 (1997), 21-27.

[6] I. Assem, S. Liu, Strongly simply connected tilted algebras, Ann. Sci. Math. Quebec 21 (1997), 13-22.

[7] I. Assem, S. LiU, Strongly simply connected algebras, Journal of algebra 207 (1998), 449-477.

[8] I. Assem, S. Liu, J. A. Peña, The strongly simple connectedness of tame tilted algebra (1998) - preprint.

[9] M. Auslander, Representation dimension of artin algebras, Queen Mary College Mathematics Notes(1971), London. 
[10] M. Auslander, I. Reiten, Representation theory of artin algebras III, Comm. Algebra, 3 (1975), 239-294.

[11] M. Auslander, I. Reiten, Representation theory of artin algebras IV, Comm. Algebra, 6 (1977), 443-518.

[12] M. Auslander, S. Smalø, Preprojective modules over artin algebras, Journal of Algebra 66 (1980), 61-122.

[13] M. Auslander, I. Reiten, S. Smalø, Representation theory of Artin algebras, Cambridge Studies in Advanced Mathematics 36 (Cambridge University Press, 1994).

[14] R. BAUtista, F. LARRIón, Auslander-Reiten quivers for certain algebras of finite representation type, J. London Math. Soc. (2), 26 (1982), 43-52.

[15] R. Bautista, F. Larrión, L. Salmerón, On Simply connected algebras, J. London Math. Soc.(2) 27 (1983), 212-220.

[16] K. Bongartz, P. Gabriel, Covering spaces in representation theory, Invent. Math., 65 (1982), 331-378.

[17] K. BongaRTz, A criterion for finite representation type, Math. Ann 269 (1984), 1-12.

[18] O.Bretscher, P. GaBriel, The standart form of a representation-finite algebra, Bull. Soc. Math. France 111 (1983), 21-40.

[19] R.-O. Buchweitz, S. LiU, Artin algebras with loops but no outer derivationspreprint.

[20] C. Cibilis, F. Larrión, L. Salmerón, Métodos diagramáticos em teoria de representações, Monografias del Instituto de Matemáticas. UNAM. México, 1982.

[21] F. U. Coelho, A Generalization of Todorov's theorem on preprojective partitions, Comm. in Algebra, 18(5) (1990), 1401-1423.

[22] F. U. Coelho, Uma introdução à teoria de representações de álgebras, Atas da XII Escola de Álgebra. Brasil, (1992). 
[23] F. U. Coelho, D. Happel, Quasitilted algebras admit a preprojective component, Proc. of Amer. Math. Soc. 125 (1997), 1283-1291.

[24] F. U. Coelho, R. R. S. VARGAS, Strongly simply connected mesh algebras - em preparação.

[25] P. DrÄXLER, Completely separanting algebras, J. Algebra 165 (1994), 550-565.

[26] P. DrÄxler, J. A. DE LA PEÑA, On the existence of postprojective componentes in the Auslander-Reiten quiver of an algebra, Tsukuba J. Math., Vol 2, número 2 (1996), 457-469.

[27] Y. Drozd, V. Kirichenko, Finite dimensional algebras, Springer-Verlag (1994), 249pp.

[28] P. Gabriel, Unzerlegbare Darstellungen I e II, Manuscripta Math. 6(1972), 71-103.

[29] P. Gabriel, A. V. Roiter, Representation of finite-dimensional algebras, Algebra VIII Encyclopaedia of Math. Sc. Vol. 73, (1992).

[30] S. Gastamiza, Preprojective partition and global dimension, Math. Japonica 35, 6 (1990), 1031-1033.

[31] S. Gastaminza, J.A. de la Peña, M. I. Platzeck, M. J. Redondo, S. TrepoDE, Finite dimensional algebras with vanishing Hochschild Cohomology, preprint.

[32] D.HAPpel, Hochschild cohomology of finite-dimensional algebras, Lectures Notes in Math., Vol. 1404, Springer-Verlag, (1989),pp.108-126.

[33] D. Happel, Hochschild Cohomology of Auslander Algebras, Topics in Algebra, Banach Center Publications, Vol. 26, Part 1 (1990), 303-310.

[34] D. Happel, C.M. Ringuel, Tilted algebras, Trans. Amer. Math. Soc. 274 (1982), 399-443.

[35] G. Hochschild, On the cohomology groups of an associative algebras, Ann. of Math. 46 (1946), 58-67. 
[36] R. Martinez-Villa, J. A. DE LA PEÑA, The Universal cover of a quiver with relations, J. Pure Appl. Algebra 30 (1983), 277-292.

[37] M. I. R. Martins, J. A. DE LA Peña, Comparing the simplicial and the Hochschild cohomologies of an algebra, a aparecer no J. Pure Applied Alg.

[38] J.A. DE LA PEÑA, A. SKowrońSki, Forbidden subcategories of non-polinomial growth tame simply connected algebras, Canad. J. Math. 48,No.5 (1995), 1018-1043.

[39] C.M. Ringel, Report on the Brauer-Thrall conjecture in Representation Theory I, Lectures Notes in Math., Vol. 831, Springer-Verlag, Berlin (1980), 104-136.

[40] C.M. Ringel, Tame algebras and integral quadratic forms, Lecture Notes in Mathematics, Vol. 1099, Springer, Berlin (1984).

[41] A.Skowroński, Algebras of polynomial growth, Topics in Algebra, Banach Center Publications, Vol. 26, Part 1 (1990), 535-568.

[42] A. Skowroński, Simply Connected Algebras and Hochschild Cohomologies, Proc. ICRA IV ( Ottawa, 1992) Can. Math. Soc. Proc., Vol. 14(1993), 431-447.

[43] A. Skowroński, Tame algebras with simply connected Galois coverings, preprint, Nicholas Copernicus University, Toruń, 1995.

[44] H. Strauss, The perpendicular category of a partial tilting module, J. Algebra 144 (1991), 43-66.

[45] G. Todorov, D. Zacharia, Remarks on Auslander algebras, Journal of Pure and Applied Algebra 70 (1991), 307-314. 